\title{
Either or Both Competition: A "Two- Sided" Theory of Advertising with Overlapping Viewerships
}

$\begin{array}{ccc}\text { Attila } & \text { Emilio } & \text { Markus } \\ \text { Ambrus } & \text { Calvano } & \text { Reisinger } \\ \text { Duke University } & \text { CREI } & \text { WHU - Otto } \\ & & \text { Beisheim } \\ & & \text { School of } \\ & & \text { Management }\end{array}$

September 1, 2014

ERID Working Paper Number 170

This paper can be downloaded without charge from the Social Science Research Network Electronic Paper Collection:

\section{Economic Research Initiatives at Duke WORKING PAPERS SERIES

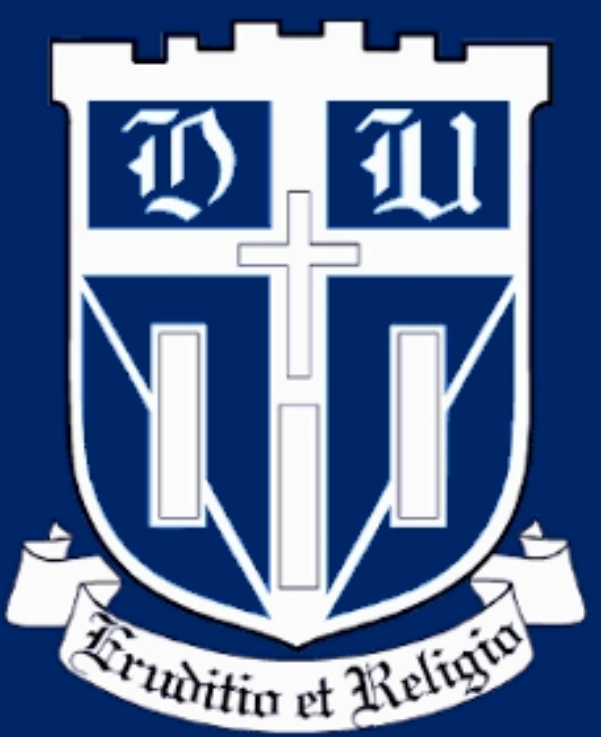




\title{
Either or Both Competition: A "Two-sided" Theory of Advertising with Overlapping Viewerships*
}

\author{
Attila Ambrus $^{\dagger}$ Emilio Calvano* Markus Reisinger $^{\S}$
}

September 2014

\begin{abstract}
In media markets, consumers spread their attention to several outlets, increasingly so as consumption migrates online. The traditional framework for studying competition among media outlets rules out this behavior by assumption. We propose a new model that allows consumers to choose multiple outlets and use it to study the effect of strategic interaction on advertising levels, and the impact of entry and mergers. We show that novel forces come into play, which reflect the outlets' incentives to control the composition of the customer base in addition to its size. We link consumer preferences and advertising technologies to market outcomes. The model can explain a number of empirical regularities that are difficult to reconcile with existing models.
\end{abstract}

Keywords: Media Competition, Two-Sided Markets, Multi-Homing, Viewer Composition, Viewer Preference Correlation

JEL-Classification: D43, L13, L82, M37

\footnotetext{
*A previous version of this paper by Ambrus and Reisinger circulated under the title "Exclusive vs. Overlapping Viewers in Media Markets". We would like to thank Simon Anderson, Elena Argentesi, Rossella Argenziano, Mark Armstrong, Susan Athey, Alessandro Bonatti, Drew Fudenberg, Matthew Gentzkow, Doh-Shin Jeon, Bruno Jullien, Marco Ottaviani, Martin Peitz, Jesse Shapiro, Gabor Virag, and Helen Weeds for helpful comments and suggestions. We would also like to thank participants at the Eleventh Annual Columbia/Duke/MIT/Northwestern IO Theory Conference, the Becker-Friedman Media Conference at Chicago Booth, the CESifo conference in Munich, the Media Workshop in Siena, the Media Markets Conference at EUI Florence, the MaCCI and ICT conference in Mannheim, and the NUS Multi-Sided Platforms Workshop, and at the Universities of Toulouse, Bergen, and Bern for their useful comments. We also thank Gina Turrini, Vivek Bhattacharya and especially Peter Landry for careful proofreading.

${ }^{\dagger}$ Department of Economics, Duke University, Durham, NC 27708. E-Mail: aa231@duke.edu

* Center for Studies in Economics and Finance, University of Naples Federico II. E-Mail: emilio.calvano@gmail.com

${ }^{\S}$ Department of Economics, WHU - Otto Beisheim School of Management, Burgplatz 2, 56179 Vallendar, Germany. E-Mail: markus.reisinger@whu.edu
} 


\section{Introduction}

A central question in the ongoing debate about the changing media landscape is how competitive forces shape advertising levels and revenues and hence assist in achieving a number of long-standing public interest goals such as enhancing entry and diversity of content. In media markets, outlets fight for consumer attention and for the accompanying stream of advertising revenues. Online advertising networks, such as the Google and Yahoo ad-networks, and traditional broadcasting stations, such as CNN and Fox News, are among the most prominent examples.

The traditional approach in media economics posits that consumers stick to the outlet they like best (for example, Anderson and Coate, 2005). So, if anything, consumers choose either one outlet or some other. Competition is for exclusive consumers as all outlets are restricted a priori to be perfect substitutes at the individual level. While compelling, this approach fails to account for the fact that many consumers satisfy their content needs on multiple outlets. This is increasingly so as content moves from paper and $\mathrm{TV}$ towards the Internet. In fact, many contend that a distinguishing feature of online consumption is the users' increased tendency to spread their attention across a wide array of outlets. Table 1 shows the reach of the six largest online advertising networks, that is, the fraction of the U.S. Internet users who, over the course of December 2012, visited a website belonging to a given network. This table shows that while Google can potentially deliver an advertising message to $93.9 \%$ of all Internet users, even the smallest of the six networks (run by Yahoo!), can deliver a whopping 83.3\%. The table highlights a key feature of these markets: different outlets provide advertisers alternate means of reaching the same users.

\begin{tabular}{|c|l|r|r|}
\hline Rank & \multicolumn{1}{|c|}{ Property } & $\begin{array}{c}\text { Unique } \\
\text { Visitors } \\
(\mathbf{0 0 0 )}\end{array}$ & $\begin{array}{c}\text { \% } \\
\text { Reach }\end{array}$ \\
\hline & $\begin{array}{l}\text { Total Internet }: \text { Total } \\
\text { Audience }\end{array}$ & 221,486 & 100.0 \\
\hline 1 & Google Ad Network $^{* *}$ & 208,074 & 93.9 \\
\hline 2 & Specific Media** $^{* *}$ & 198,119 & 89.5 \\
\hline 3 & $\begin{array}{l}\text { Federated Media Publisher } \\
\text { Network }^{* *}\end{array}$ & 193,453 & 87.3 \\
\hline 4 & AOL Advertising $^{* *}$ & 186,595 & 84.2 \\
\hline 5 & AT\&T AdWorks $^{* *}$ & 185,757 & 83.9 \\
\hline 6 & Genome from Yahoo!** & 184,556 & 83.3 \\
\hline
\end{tabular}

Table 1. Top 6 Online Ad Networks by reach.

(Source: Comscore press release: "ComScore MMX Ranks Top 50 U.S.

Web Properties for December 2012," 28th January 2013)

Motivated by these observations, the paper has two goals. First, we propose an alternative model of competition that replaces the assumption of perfect substitutability by allowing consumers to access content on multiple outlets. So, with two outlets, consumers can choose either one outlet or both (or none). Specifically, we work under the (extreme) assumption that consumer demand for one outlet does not affect the demand for another outlet. This is what we call either or both competition in contrast with the standard framework discussed above. We claim that this model of competition is an appealing alternative to existing ones for several reasons. It is a good approximation of reality in some non-trivial contexts where substitutability is limited. For example, choosing Facebook.com for online social networking services is arguably orthogonal to choosing Yelp.com as one's supplier of restaurant 
reviews. ${ }^{1}$ Moreover, and somewhat surprisingly, Gentzkow, Shapiro and Sinkinson (2014) document limited substitutability even in traditional media markets such as that of U.S. newspapers. They show that on average $86 \%$ of an entrant's circulation comes from households reading multiple newspapers or households who previously did not read at all.

Second, we apply the model to study the market provision of advertising opportunities, which has been the focus of a large literature in media markets. For instance, does increased competition between media outlets reduce the amount of ads? Is competition weakened if outlets supply diverse content? For example, should we expect the impact of entry of Fox News on MSNBC's choices to be different than that of Fox Sports on ESPN's choices? ${ }^{2}$ We propose a characterization of the incentives to provide advertising opportunities in duopoly and draw implications for the equilibrium advertising levels and prices and for the impacts of entry and mergers. We link consumer preferences and advertising technologies to market outcomes and derive results in terms of empirical objects.

The baseline model features two outlets, with continuums of consumers and advertisers. Consumers "dislike" ads in the sense that they would rather get ad-free content. ${ }^{3}$ Advertisers want to reach more consumers, as greater consumer exposure increases demand for their products. Outlets choose the quantity of ads striving to strike the right balance. The ads are allocated to advertisers according to a simple contracting environment in which each outlet offers a contract specifying a price for a given advertising intensity.

We do not impose a specific functional form on either the distribution of consumer preferences or on the advertising technology. However, in our baseline model we make the simplifying assumption that advertisers are homogeneous. This formulation allows us to address questions that are not tractable in the traditional framework, such as how consumer preference correlation affects equilibrium advertising levels, and how it influences the effect of outlet entry. ${ }^{4}$ In addition, we provide a natural generalization that allows for heterogeneous advertisers, and extend some results to this setting.

A key component of our model is that consumers who are exclusive to an outlet are more valuable than overlapping (also called "multi-homing") consumers. As the latter are catered to by more than one outlet, no individual outlet can extract from the advertisers more than the incremental value of reaching these same consumers via an additional outlet. This implies that in our model outlets do not only care about the overall consumer demand level, as in existing models, but also about its composition, i.e., the fraction of exclusive versus overlapping consumers. ${ }^{5}$ Indeed, it is common for ad networks to assess the

\footnotetext{
${ }^{1}$ According to the source supra cited, Facebook and Yelp are among the top 10 most-visited U.S. websites and in fact belong to different advertising networks.

${ }^{2}$ Fox News has, arguably, a conservative bias, so it is unlikely to appeal to MSNBC's core liberal viewers. In contrast, Fox Sports, arguably, caters to the same preferences as ESPN.

${ }^{3}$ This assumption could be relaxed, in that we could allow for positive marginal utility of advertisements at low levels of advertising, as long as advertisements ultimately become a nuisance. In equilibrium, outlets would always raise advertising levels to this range.

${ }^{4}$ Existing models either assume a Hotelling framework, imposing perfect negative correlation in consumer preferences for two outlets, or a representative consumer framework. In contrast, our framework allows for consumer preferences to be correlated in any way between outlets.

${ }^{5}$ That multi-homing consumers are worth less to advertisers is consistent with the well-documented fact in the television industry that the per-viewer fee of an advertisement on programs with more viewers is larger. In the U.S., for instance Fisher, McGowan and Evans (1980) find this regularity. Our model accounts for this since reaching the same number of eyeball pairs through broadcasting a commercial to a large audience implies reaching more viewers than reaching the same
} 
extent of overlap and for advertisers to take into account the extent of duplication in large cross-outlet campaigns. To stress the importance of these considerations, we document ${ }^{6}$ Google's sales pitch for its Display Network (GDN). It employs proprietary data to assess the effect of an advertising campaign on auto insurance. The sales pitch emphasizes that a "key takeaway" is that the GDN "exclusively reaches $30 \%$ of the auto-insurance seekers" that do not visit Yahoo, $36 \%$ that do not visit Youtube and so on.

Characterizing the equilibrium choices of the outlets, we find that accounting for multi-homing changes the nature of competition substantially. In particular, we show that two novel forces come into play when some consumers are shared. In duopoly, multi-homers receive advertising messages from two different sources. This fact, together with diminishing returns from advertising, implies that the marginal ad is less valuable than it would be in monopoly. This duplication effect induces outlets, among other things, to supply fewer ads. Second, as discussed above, in duopoly common consumers are of lower value. So the opportunity cost, in terms of lost consumers, of increasing the advertising level is lower relative to that of a monopolist whose consumers are all exclusive by definition. As a result, duopolists are more aggressive in the sense that they are less wary of increases in advertising level. This business-sharing effect induces higher equilibrium advertising levels. We provide an intuitive and full characterization of how the novel effects interact and shape equilibrium outcomes, in terms of the elasticities of consumer demand and of the properties of the advertising technology.

To understand under what conditions either effect prevails, we trace out the impact of competition to two sources: a preference-driven and a technology-driven source.

On the preference side, the key question turns out to be: are overlapping consumers more responsive to changes in the advertising level relative to exclusive ones? If yes, then the business-sharing effect dominates. A follow-up question is: when is it the case that increasing the amount of advertising disproportionately repels overlapping consumers? To illustrate our results, consider the preference correlation of consumers for media outlets. For example, suppose Fox News were to enter, MSNBC being the incumbent. As these stations do not share the same ideological affiliation, one can reasonably conjecture that viewer preferences are negatively correlated. This implies that a large portion of viewers of each outlet will be exclusive. When reducing the advertising level, an outlet attracts more viewers, both single-homers and multi-homers. Compared to its viewer base, these marginal viewers are comprised of a larger portion of multi-homers. Since multi-homers are less valuable, incentives to lower advertising are small, and equilibrium advertising levels are high - the business-sharing effect. Conversely, if the viewer preference correlation is positive, for example with Fox Sports Channel and ESPN, advertising levels fall with entry. We provide a first empirical pass using data from the U.S. cable TV industry that provides suggestive evidence for these results.

On the technology side, instead, the key question is: is the elasticity of informing exclusive consumers higher than the one of informing overlapping consumers? If yes, the duplication effect prevails. The intuition for this result is as follows: Since overlapping consumers may spend a smaller amount of attention on each outlet than exclusive ones, an ad to an exclusive consumer is more likely to be successful. Everything else constant, informing exclusive consumers is therefore more effective, which leads to a

number of eyeball pairs through a series of commercials to smaller audiences, because the latter audiences might have some viewers in common. See Ozga (1960) for an early observation of this fact.

${ }^{6}$ See Figure 2 in Appendix 11.4. 
reduction of advertising in duopoly. At the same time, due to the smaller amount of attention, the probability that an overlapping consumer becomes informed by an ad is lower. Coupled with diminishing returns from advertising, this may render advertising to multi-homing consumers more beneficial, thereby inducing a countervailing force. We show that the latter effect dominates in the case when the advertising technology takes the widely used exponential form.

We also show that the model can account for a number of empirical regularities which are deemed puzzling under the traditional approach. For example, there is evidence of advertising levels rising with competition (e.g., the so-called "Fox News Puzzle," documented in the appendix, is one well known illustration). ${ }^{7}$ In the traditional approach with perfect substitutability at the consumer level, the amount of advertising supplied by outlets can be considered as an implicit price for consumers. Competition typically lowers prices as outlets try to woo consumers from their rivals - the familiar business-stealing effect. Therefore, increased competition always reduces advertising levels.

On the policy side, we consider the effect of outlet mergers on market outcomes. We show that if advertising to exclusive and overlapping consumers is equally effective, a merger does not change the equilibrium advertising levels. Intuitively, when an outlet in duopoly raises its advertising level, some overlapping consumers no longer connect to the outlet. The outlet then loses the incremental value that it can extract from advertisers for reaching these consumers. By contrast, an owner of both outlets can extract the full value from advertisers. However, when raising the advertising level on an outlet, some overlapping consumers become exclusive ones on the other outlet. We show that the lost profit incurred by the owner of both outlets is exactly equal to the incremental value that a duopolist loses, implying that the trade-off in both scenarios is the same. ${ }^{8}$ This result is important both for economic theory and policy discussion as it shows that mergers in media markets can be neutral with respect to social welfare.

Classic contributions in media economics, for example, Spence and Owen (1977) or Wildman and Owen (1985), impose perfect substitutability and do not allow for endogenous advertising levels or twosided externalities between viewers and advertisers. More recently, the seminal contribution of Anderson and Coate (2005) explicitly accounts for these externalities. ${ }^{9}$ In their model, viewers are distributed on a Hotelling line with outlets located at the endpoints. Similar to early works, viewers watch only one channel while advertisers can buy commercials on both channels. In this setting, Anderson and Coate (2005) show, among several other results, that competitive pressure reduces the amount of ads and that this reduction is not necessarily socially desirable. The framework with single-homing viewers has been used to tackle a wide array of questions. ${ }^{10}$

\footnotetext{
${ }^{7}$ Several observers contend that the wave of channel entry during the 1990s in the U.S. cable TV industry coincided with an increase in advertising levels on many channels (this is what is usually referred to as the Fox News puzzle). In Appendix 11.3, using a dataset provided by Kagan-SNL, we show that an increase in the number of U.S. cable TV channels is on average indeed associated with an increase in advertising levels on incumbent channels.

${ }^{8}$ This result is reminiscent of common agency models (e.g., Bernheim and Whinston, 1986) that obtain equivalent allocations in competition and cooperation. However, the mechanism in our model differs from the one in common agency frameworks.

${ }^{9}$ For different applications of such two-sided market models, see e.g., Armstrong (2006), Ellison and Fudenberg (2003), and Rochet and Tirole (2003).

${ }^{10}$ For example, Armstrong (1999) and Harbord and Ottaviani (2001) consider exclusivity arrangements for premium content in pay-TV markets, Dukes and Gal-Or (2003) study product market competition between advertisers, Peitz and Valletti (2008) analyze optimal locations of stations, Crampes, Haritchabalet and Jullien (2009) consider the effects of free entry of outlets, and Anderson and Peitz (2012) allow advertising congestion. Kind, Nilssen and Sørgard (2009) consider a
} 
There are a few recent studies that propose models to study competition in media markets that account for multi-homing. Anderson, Foros and Kind (2014) consider a model similar in spirit to ours. In contrast to our paper, the equilibrium concept they employ (passive instead of responsive expectations) implies that outlets cannot attract consumers via lower ad-levels. As a consequence, the novel effects identified here are not present. ${ }^{11}$ Instead, they analyze public broadcasting and reconsider the classic Steiner (1952) result. Athey, Calvano and Gans (2013) and Bergemann and Bonatti (2011, in Sections 5 and 6) also consider multi-homing viewers but are mainly concerned with different tracking/targeting technologies and do not allow for advertisements generating (negative) externalities on viewers, which is at the core of our model. ${ }^{12}$

On the empirical side, Gentzkow, Shapiro and Sinkinson (2014) develop a structural model of the newspaper industry that applies our finding that advertising-market competition depends on the extent of overlap in readership. They find that competition increases diversity significantly, offsetting the incentive to cater to the tastes of majority consumers (George and Waldfogel, 2003). ${ }^{13}$

The rest of the paper is organized as follows: Section 2 introduces the model and Section 3 presents some preliminary analysis. Section 4 analyzes outlet competition and presents the main trade-offs of our model. Section 5 considers the effects of viewer preference correlation and Section 6 explores the advertising technology. Sections 7 considers outlet mergers. Section 8 analyzes welfare implications and Section 9 presents an extension to heterogeneous advertisers. Section 10 concludes. All proofs can be found in the Appendix.

\section{$2 \quad$ The Model}

The basic model features a unit mass of heterogeneous viewers, a unit mass of homogeneous advertisers and two outlets indexed by $i \in\{1,2\} .{ }^{14}$

\section{Viewer Demand}

Viewers are parametrized by their reservation utilities $\left(q_{1}, q_{2}\right) \in \mathbb{R}^{2}$ for outlets 1 and 2 , where $\left(q_{1}, q_{2}\right)$ is distributed according to a bivariate probability distribution with smooth joint density denoted $h\left(q_{1}, q_{2}\right)$. A viewer of $\left(q_{1}, q_{2}\right)$-type joins outlet $i$ if and only if $q_{i}-\gamma n_{i} \geq 0$, where $n_{i}$ is the advertising level on outlet $i$ and $\gamma>0$ is a nuisance parameter. Given the advertising level on each outlet, we can back out the demand system:

representative viewer framework and analyze the efficiency of the market equilibrium with respect to the advertising level and allow for viewer payments.

${ }^{11}$ In this respect, our analysis is in line with the canonical two-sided market framework, in which a change in the quantity on one side changes the outlet's attractiveness on the other side.

${ }^{12}$ Rüdiger (2013) applies the idea that multi-homers are worth less to study the implications of "cross checking" on mediabias. He finds that diminishing returns to scale from advertising increase incentives of outlets to move towards extreme positions.

${ }^{13}$ Sweeting (2013) provides a rich dynamic structural framework that allows to back out the drivers of product variety in the commercial radio industry under the assumption that listeners single-home. In light of our theory, an open question is how business sharing considerations affect the results.

${ }^{14}$ We cast our model in terms of the television context. The model also applies to internet or radio, where the term viewers would be replaced by users or listeners. 
Multi-homers: $\quad D_{12}:=\operatorname{Prob}\left\{q_{1}-\gamma n_{1} \geq 0 ; q_{2}-\gamma n_{2} \geq 0\right\}$,

Single-homers $1: \quad D_{1}:=\operatorname{Prob}\left\{q_{1}-\gamma n_{1} \geq 0 ; q_{2}-\gamma n_{2}<0\right\}$,

Single-homers $2: \quad D_{2}:=\operatorname{Prob}\left\{q_{1}-\gamma n_{1}<0 ; q_{2}-\gamma n_{2} \geq 0\right\}$,

Zero-homers: $\quad D_{0}:=1-D_{1}-D_{2}-D_{12}$.

The demand system is discussed at length at the end of this section. To ensure uniqueness of the equilibrium and interior solutions, we assume that demand functions are well-behaved. This boils down to assumptions on the joint density function $h\left(q_{1}, q_{2}\right)$. However, it is not necessary to spell out assumptions on this function, since we will later only work with the resulting demand functions. ${ }^{15}$

\section{Timing and Outlets' Choices}

Outlets compete for viewers and for advertisers. Outlets receive payments only from advertisers but not from viewers. To make the model as transparent as possible, we develop a four-stage game. When discussing the modeling assumptions, we relate this model's equilibrium outcome to a canonical two-stage model of platform competition à la Armstrong (2006).

In stage 1 , outlets simultaneously set the total advertising levels $n_{1}$ and $n_{2}$. In stage 2 , viewers observe $n_{1}$ and $n_{2}$ and choose which outlet(s) to join, if any. In stage 3, outlets simultaneously offer menus of contracts to advertisers. A contract offered by outlet $i$ is a pair $\left(t_{i}, m_{i}\right) \in \mathbb{R}_{+}^{2}$, which specifies an advertising intensity $m_{i} \geq 0$ in exchange for a monetary transfer $t_{i} \geq 0$. Finally, in stage 4 , advertisers simultaneously decide which contract(s), if any, to accept. Below we will show that in our basic model with homogeneous advertisers, each outlet only offers one contract in equilibrium, and this contract is accepted by all advertisers. ${ }^{16}$ This implies that, in equilibrium, $m_{i}=n_{i}$ for the unique advertising intensity $m_{i}$ offered by outlet $i$.

To ensure that the announced advertising levels are consistent with the realized levels after stage 4 , we assume that if total advertising levels accepted by advertisers at outlet $i$ exceed $n_{i}$, then outlet $i$ obtains a large negative payoff. ${ }^{17}$ Therefore, our game is similar to Kreps and Scheinkman (1983), i.e., in the first stage outlets choose an advertising level that puts an upper bound on the advertising intensities they can sell subsequently.

The extensive form captures actual practice in U.S. and Canadian broadcasting markets. On a seasonal basis, broadcasters and advertisers meet at an "upfront" event to sell commercials on the networks' upcoming programs. At this point the networks' supply of commercial breaks is already determined. Also, the Nielsen rating system, which measures viewership for different programs and outlets (and ad-

${ }^{15}$ For example, sufficient (but not necessary) assumptions to obtain a unique interior solution are

$$
\frac{\partial^{2} D_{i}}{\partial n_{i}^{2}} \leq 0, \quad \frac{\partial^{2} D_{12}}{\partial n_{i}{ }^{2}} \leq 0 \quad \text { and } \quad\left|\frac{\partial^{2} D_{i}}{\partial n_{i}{ }^{2}}\right| \geq\left|\frac{\partial^{2} D_{i}}{\partial n_{i} \partial n_{j}}\right|, \forall i=1,2 \text { and } j=3-i .
$$

See e.g., Vives (2000) for a detailed discussion of why these assumptions ensure concavity of the objective functions and uniqueness of the equilibrium.

\footnotetext{
${ }^{16}$ In Section 9 we consider a model with heterogeneous advertisers in which outlets do offer multiple contracts in equilibrium.

${ }^{17}$ Our results would remain unchanged if we instead assume that actual advertising intensities are rationed proportionally for participating advertisers in case there is excess demand for an outlet's advertising intensities. We stick to the current formulation as it simplifies some of the arguments in the proofs.
} 
vertisers) supplies viewership estimates. Contracts that specify, among other things, the number of ads (so-called "avails") in exchange for a fixed payment are then signed between broadcasters and advertisers.

The solution concept we use throughout the paper is subgame perfect Nash equilibrium (SPNE).

\section{Advertising Technology}

Advertising in our model is informative. We normalize the return of informing a viewer about a product to $1 .^{18}$ In line with the literature, e.g., Anderson and Coate (2005) or Crampes, Haritchabalet and Jullien (2009), we assume that advertisers can fully extract the value of being informed from the consumers.

The mass of informed viewers (also known as "reach") is determined by the number of advertising messages $\left(m_{1}, m_{2}\right)$ a particular advertiser purchases on each outlet. Without loss of generality, we decompose the total reach as the sum of the reach within the three different viewers' subsets. We denote the probability with which a single-homing viewer on outlet $i$ becomes informed of an advertiser's product by $\phi_{i}\left(m_{i}\right)$. We assume that $\phi_{i}$ is smooth, strictly increasing and strictly concave, with $\phi_{i}(0)=0$. That is, there are positive but diminishing returns to advertising.

By definition, $\phi_{12}$ equals the probability that a multi-homing viewer becomes informed on some outlet. In what follows, we decompose $\phi_{12}$ as one minus the probability that the viewer is not informed on either outlet, that is, $\phi_{12}\left(m_{1}, m_{2}\right):=1-\left(1-\hat{\phi}_{1}\left(m_{1}\right)\right)\left(1-\hat{\phi}_{2}\left(m_{2}\right)\right)$, where $\hat{\phi}_{i}\left(m_{i}\right)$ is the probability that an overlapping viewer becomes informed on outlet $i$, where $\hat{\phi}_{i}\left(m_{i}\right)$ is also smooth, strictly increasing and strictly concave. We assume (omitting arguments) that $\phi_{i} \geq \hat{\phi}_{i}$ and $\partial \phi_{i} / \partial m_{i} \geq \partial \hat{\phi}_{i} / \partial m_{i}$, that is, the probability of informing a single-homing viewer through a given outlet is (weakly) larger than the probability of informing a multi-homing viewer, given advertising intensity $m_{i}$. This also holds for the marginal probability. We provide a detailed discussion of this assumption below.

We note that $\phi_{i}$ and $\hat{\phi}_{i}$ only depend on $m_{i}$. Therefore, $\partial \phi_{i} / \partial m_{j}=0$ and $\partial \hat{\phi}_{i} / \partial m_{j}=0$. The first equation implies the natural property that the probability of informing an exclusive viewer of outlet $i$ does not depend on the advertising intensity on outlet $j$. However, the second equation does not imply that the probability of informing an overlapping viewer does not depend on $m_{j}$. (In fact, from the definition of $\phi_{12}$, we have $\partial \phi_{12} / \partial m_{j}=\partial \phi_{j} / \partial m_{j}\left(1-\hat{\phi}_{i}\left(m_{i}\right)\right)>0$.) It only implies that the probability of informing a multi-homer on a particular outlet is independent of the advertising intensity on the rival outlet. Note also that $\phi_{i}, \hat{\phi}_{i}, \phi_{j}$ and $\hat{\phi}_{j}$ may all be different.

\section{Payoffs}

An outlet's payoff is equal to the total amount of transfers it receives (for simplicity, we assume that the marginal cost of ads is zero). An advertiser's payoff, in case he is active on both outlets, is $u\left(n_{1}, n_{2}, m_{1}, m_{2}\right)-t_{1}-t_{2}$, where

$$
u\left(n_{1}, n_{2}, m_{1}, m_{2}\right):=D_{1}\left(n_{1}, n_{2}\right) \phi_{1}\left(m_{1}\right)+D_{2}\left(n_{1}, n_{2}\right) \phi_{2}\left(m_{2}\right)+D_{12}\left(n_{1}, n_{2}\right) \phi_{12}\left(m_{1}, m_{2}\right)
$$

and $t_{1}$ and $t_{2}$ are the transfers to outlets 1 and 2 , respectively. If he only joins outlet $i$, the payoff is $u\left(n_{i}, n_{j}, m_{i}, 0\right)-t_{i}=D_{i}\left(n_{i}, n_{j}\right) \phi_{i}\left(m_{i}\right)+D_{12}\left(n_{i}, n_{j}\right) \hat{\phi}_{i}\left(m_{i}\right)-t_{i}$ since the advertiser reaches viewers only

\footnotetext{
${ }^{18}$ In Section 9, we allow advertisers to be different with respect to this return.
} 
via outlet $i$. Advertisers' reservation utilities are normalized to zero.

\section{Discussion of Modeling Choices}

Conditional on the realization of the utility parameters $\left(q_{1}, q_{2}\right)$, a viewer's choice of whether to join outlet $i$ is assumed to depend neither on $n_{j}$ nor on $q_{j}$. This 'demand independence' assumption should not be confused with nor does it imply statistical independence between $q_{i}$ and $q_{j}$. For instance, the model allows preferences for $i$ (say Facebook) and $j$ (say Yelp) to be correlated to account for some underlying common covariate factor (say 'internet savviness'). In fact, the model nests those specifications which add structure to preferences by positing a positive or negative relationship between valuations of different outlets. An extreme example is the Hotelling-type spatial model with the two outlets at the opposite ends of a unit interval and viewers distributed along the interval. Thus, the Hotelling specification could be captured by the above setup with the restriction $q_{1}=1-q_{2}$.

An important property of the demand schedules, following directly from the way we defined them, is that if $n_{i}$ changes but $n_{j}$ is unchanged, the choice of whether to join outlet $j$ remains unaffected. This property contrasts formulations in which viewers choose one outlet over the other. In our framework, if $n_{i}$ increases, outlet $i$ loses some single-homers and some multi-homers. The single-homers become zero-homers while the multi-homers become single-homers on outlet $j$. The latter effect implies that $\partial D_{12} / \partial n_{i}=-\partial D_{j} / \partial n_{i}$.

The $\phi$-functions capture, in a parsimonious way, several relevant aspects of consumer behavior, outlet asymmetry, and advertising technology. For example, if one outlet is more effective at reaching viewers for all nonzero advertising levels, or if viewers spend more time on one outlet than on the other, this could be captured by the restriction $\phi_{i}(m)>\phi_{j}(m)$ for all $m>0$. The assumption $\hat{\phi}_{i}(m) \leq \phi_{i}(m)$ allows us to capture heterogeneity in behavior across viewer sets. While we are agnostic here as to the source of this heterogeneity, an important motivation is that it accounts for multi-homers spending a reduced amount of attention on a particular outlet. If the return from an additional unit of time spent on either outlet is decreasing, it is natural that multi-homers spread this limited time across outlets so that $\hat{\phi}_{i}(m)<\phi_{i}(m)$ for all $m$. The assumption $\partial \phi_{i} / \partial m_{i} \geq \partial \hat{\phi}_{i} / \partial m_{i}$ captures the natural property that the marginal return of advertising is larger for exclusive than for overlapping viewers, that is, $\partial \phi_{i} / \partial m_{i}>\partial \phi_{12} / \partial m_{i}$. To see this, note that $\partial \phi_{i} / \partial m_{i}>\partial \hat{\phi}_{i} / \partial m_{i}\left(1-\hat{\phi}_{j}\left(m_{j}\right)\right)=\partial \phi_{12} / \partial m_{i}$, where the inequality follows from the assumption $\partial \phi_{i} / \partial m_{i} \geq \partial \hat{\phi}_{i} / \partial m_{i}$ and $\hat{\phi}_{j}\left(m_{j}\right)>0$.

The game presented is equivalent to a three-stage game whereby advertisers and viewers simultaneously make their choices. In turn, we show in Appendix 11.2 that this game is equivalent (with one caveat) to a two-stage duopoly model in which outlets simultaneously make offers and, upon observing the offers, all agents simultaneously make their choices. The role of stage 1 in our model is to relax the dependence of viewers' choices on advertisers' choices. Indeed, viewerships are fixed before outlets sell their advertising slots. The assumption that the aggregate advertising level is fixed at the contracting stage greatly simplifies the analysis. In Appendix 11.2 we relax it by considering a version of the model in which outlets do not announce total advertising levels, but instead offer contracts of the form $\left(t_{i}, m_{i}\right)$ to advertisers, and afterwards viewers and advertisers simultaneously decide which outlet to join. We show that under some additional conditions on preferences, there exists an outcome-equivalent SPNE to that of our game. This two-stage game is much harder to analyze since a deviation by one outlet leads to simultaneous changes in viewers' and advertisers' decisions that are influenced by each other. For this reason, and due to the outcome-equivalence under certain conditions, we stick to the easier formulation. 


\section{Preliminaries: Contracting Stage}

To identify the competitive forces, we proceed by contrasting the market outcome of the game just described, in which two outlets compete, with the monopoly case, in which only one outlet is present in the market. We first solve the contracting stage.

A key observation is that after any pair of first stage announcements $\left(n_{1}, n_{2}\right)$, in any continuation equilibrium, outlets spread their advertising level equally across all advertisers. This result follows due to diminishing returns from advertising. As there is a unit mass of advertisers, the number of advertising intensities offered to each advertiser by outlet $i$ is equal to $n_{i}$. In turn, the equilibrium transfer is the incremental value that advertising intensity $n_{i}$ on outlet $i$ generates for an advertiser who already advertises with intensity $n_{j}$ on the other outlet. ${ }^{19}$

Claim 1: In any SPNE of a game with competing outlets, given any pair of first-stage choices $\left(n_{1}, n_{2}\right)$, each outlet $i$ only offers one contract $\left(t_{i}, m_{i}\right)$. These contracts are accepted by all advertisers, and have the feature that $m_{1}=n_{1}, m_{2}=n_{2}, t_{1}=u\left(n_{1}, n_{2}\right)-u\left(0, n_{2}\right)$ and $t_{2}=u\left(n_{1}, n_{2}\right)-u\left(n_{1}, 0\right)$.

The next claim establishes a parallel result for the single outlet (that is, monopoly) case, whose proof we omit because it proceeds along the same lines as the proof of Claim 1 above. In particular the monopolist offers a single contract that is accepted by all advertisers.

Claim 2: In any SPNE of a game with a monopolistic outlet, given first-stage choice $n_{i}$, the monopolist offers a single contract $\left(t, m_{i}\right)$. This contract is accepted by all advertisers, and has the feature that $m_{i}=n_{i}$, and $t=u\left(n_{i}, 0\right)$.

In what follows, we denote $D_{i}\left(n_{1}, n_{2}\right)+D_{12}\left(n_{1}, n_{2}\right)$ by $d_{i}\left(n_{i}\right)$, that is, $d_{i}\left(n_{i}\right):=\operatorname{Prob}\left\{q_{i}-\gamma n_{i} \geq 0\right\}$. Claims 1 and 2 imply that, because in equilibrium viewers correctly anticipate the unique continuation play following stage 1 , in any SPNE viewer demand on outlet $i$ is $d_{i}\left(n_{i}\right), i=1,2$. Furthermore, outlets' equilibrium profits in duopoly are lower than the equilibrium profit obtained by the monopolist. In duopoly, outlets can only demand the incremental value from an advertiser who is also active on the other outlet, whereas a monopolist can extract the whole surplus. Specifically, a monopolist outlet $i$ obtains a profit of $d_{i}\left(n_{i}\right) \phi_{i}\left(n_{i}\right)$, since it has only exclusive viewers, whereas outlet $i$ in duopoly only obtains $D_{i}\left(n_{i}\right) \phi_{i}\left(n_{i}\right)+D_{12}\left(n_{1}, n_{2}\right)\left(\phi_{12}\left(n_{1}, n_{2}\right)-\hat{\phi}_{j}\left(n_{j}\right)\right)$, because it shares some viewers with its rival.

\section{Outlet Competition}

We proceed by contrasting the choice of a monopolist ${ }^{20}$

$$
n_{i}^{m}:=\arg \max _{n_{i}} \quad d_{i}\left(n_{i}\right) \phi_{i}\left(n_{i}\right)
$$

\footnotetext{
${ }^{19}$ With a slight abuse of notation, in what follows, we denote $u\left(n_{i}, n_{j}, n_{i}, n_{j}\right)$ by $u\left(n_{i}, n_{j}\right)$ and $u\left(n_{i}, n_{j}, n_{i}, 0\right)$ by $u\left(n_{i}, 0\right)$.

${ }^{20}$ Here we adopt the convention that $i$ denotes the monopoly outlet.
} 
with the duopoly outcome, that is, with the fixed point of the best reply correspondences

$$
n_{i}^{d}:=\arg \max _{n_{i}} \quad D_{i}\left(n_{i}\right) \phi_{i}\left(n_{i}\right)+D_{12}\left(n_{1}, n_{2}\right)\left(\phi_{12}\left(n_{1}, n_{2}\right)-\hat{\phi}_{j}\left(n_{j}\right)\right) \quad i=1,2 ; \quad j=3-i .
$$

Our goal is to determine the effects that drive competition in this model. For this purpose, it is useful to rewrite the duopolist's profit as if all viewers were exclusive plus a correction term that accounts for the fact that outlet $i$ can only extract the incremental value from its shared viewers:

$$
n_{i}^{d}:=\arg \max _{n_{i}} d_{i}\left(n_{i}\right) \phi_{i}\left(n_{i}\right)+D_{12}\left(n_{1}, n_{2}\right)\left(\phi_{12}\left(n_{1}, n_{2}\right)-\hat{\phi}_{j}\left(n_{j}\right)-\phi_{i}\left(n_{i}\right)\right) .
$$

First consider problem (2). Its solution is characterized by the first-order condition

$$
\frac{\partial \phi_{i}}{\partial n_{i}} d_{i}+\frac{d d_{i}}{d n_{i}} \phi_{i}=0
$$

When increasing $n_{i}$, outlet $i$ trades off profits on inframarginal viewers due to increased reach with profits on marginal viewers who switch off. If we introduce the advertising elasticities of the total demand $d_{i}$ and of the advertising function $\phi_{i}$ with respect to $n_{i}$,

$$
\eta_{d_{i}}:=-\frac{\partial d_{i}}{\partial n_{i}} \frac{n_{i}}{d_{i}} \quad \text { and } \quad \eta_{\phi_{i}}:=\frac{\partial \phi_{i}}{\partial n_{i}} \frac{n_{i}}{\phi_{i}}
$$

the optimal advertising level is characterized by the simple and intuitive condition

$$
\eta_{\phi_{i}}=\eta_{d_{i}}
$$

Consider now problem (4). In duopoly, condition (5) is augmented to account for the fact that some of the previously exclusive viewers are now shared. The first-order condition can be written as

$$
\frac{\partial \phi_{i}}{\partial n_{i}} d_{i}+\frac{\partial d_{i}}{\partial n_{i}} \phi_{i}+D_{12} \frac{\partial\left(\phi_{12}-\hat{\phi}_{j}-\phi_{i}\right)}{\partial n_{i}}+\frac{\partial D_{12}}{\partial n_{i}}\left(\phi_{12}-\hat{\phi}_{j}-\phi_{i}\right)=0
$$

To build intuition, consider the simplest case in which the two outlets are symmetric, i.e., $d_{i}(n)=$ $d_{j}(n), \phi_{i}(n)=\phi_{j}(n)$, and $\hat{\phi}_{i}(n)=\hat{\phi}_{j}(n)$ for all $n$ and suppose that competing outlets behave as the monopolist does, that is, $n_{j}^{d}=n_{i}^{d}=n_{i}^{m}$. Can these advertising levels constitute an equilibrium? First, overlapping viewers receive advertising messages from two outlets. If each outlet chooses the same advertising level as the monopolist, theamount of advertising viewers are exposed to doubles in duopoly. Other things held constant, decreasing marginal returns give an incentive to scale back advertising on outlet $i$, whose marginal contribution to the advertisers' surplus drops as a result of such duplication. This duplication effect is captured by the third term of (6). This term is negative because $\partial \phi_{i} / \partial n_{i}>$ $\partial \phi_{12} / \partial n_{i}$. The second, arguably more subtle, effect is captured by the fourth term in (6). It is positive as $\partial D_{12} / \partial n_{i}<0$ and $\phi_{12}-\hat{\phi}_{j}-\phi_{i}<0 .{ }^{21}$ For a duopolist, the total variation in demand due to a small increase in $n_{i}$ decomposes to $\partial D_{i} / \partial n_{i}$ and $\partial D_{12} / \partial n_{i}$. The first term is the change in the mass of exclusive viewers and the second the change in the mass of overlapping viewers. Instead, a monopolist has only

${ }^{21}$ The term $\phi_{12}-\hat{\phi}_{j}-\phi_{i}$ is equivalent to $\hat{\phi}_{i}-\phi_{i}-\hat{\phi}_{i} \hat{\phi}_{j}$, which is negative because $\phi_{i} \geq \hat{\phi}_{i}$ and $\hat{\phi}_{i} \hat{\phi}_{j}>0$. 
exclusive viewers and is therefore wary only of the total variation of $\partial d_{i} / \partial n_{i}{ }^{22}$ Since exclusive viewers are more valuable than overlapping viewers, in duopoly the opportunity cost of losing shared business is lower than that of losing exclusive business. Other things held constant, this business-sharing effect gives the outlet an incentive to increase its advertising levels.

Before moving on, we stress that the business-sharing effect points in the opposite direction than the one brought about by competition in traditional two-sided single-homing setups. A key insight there is that competitive pressure induces competing outlets to put more emphasis on lost business than monopolists do. (See, for example, the discussion in Armstrong (2006), Section 4). Lost business on one side would lower revenues on the other side of the market, as consumers find the rival more attractive because of the indirect network effects. As a consequence, advertising levels, which act as a price for viewers, fall if competitive pressure increases. By contrast, in our model the business-sharing effect leads to higher advertising levels. Intuitively, if multi-homing viewers are relatively more responsive than single-homing viewers, that is, if they account for a relatively high portion of the variation, losing viewers is less detrimental for the duopolist. Therefore, outlets put less emphasis on lost business in duopoly relative to monopoly, leading to the opposite result.

The following proposition provides the precise condition for advertising levels in duopoly being larger than in monopoly. Let

$$
\eta_{D_{12}}:=-\frac{\partial D_{12}}{\partial n_{i}} \frac{n_{i}}{D_{12}} \quad \text { and } \quad \eta_{\phi_{i}+\hat{\phi}_{j}-\phi_{12}}:=\frac{\partial\left(\phi_{i}+\hat{\phi}_{j}-\phi_{12}\right)}{\partial n_{i}} \frac{n_{i}}{\phi_{i}+\hat{\phi}_{j}-\phi_{12}}
$$

Proposition 1: An incumbent monopolist's advertising level increases (decreases) upon entry of a competitor if and only if

$$
\frac{\eta_{D_{12}}}{\eta_{d_{i}}}>(<) \frac{\eta_{\phi_{i}+\hat{\phi}_{j}-\phi_{12}}}{\eta_{\phi_{i}}}
$$

where all functions are evaluated at $n_{i}=n_{i}^{m}$ and $n_{j}=n_{j}^{d}$.

The left-hand side of (7) is the ratio of the demand elasticity of overlapping viewers to the demand elasticity of viewers in monopoly. To interpret the right-hand side, let us first rewrite the profit function of an outlet in duopoly. Defining $\Delta_{\phi_{i}}\left(n_{i}\right) \equiv \phi_{i}\left(n_{i}\right)-\hat{\phi}_{i}\left(n_{i}\right) \geq 0$ and plugging in $\phi_{12}=\hat{\phi}_{1}\left(n_{1}\right)+\hat{\phi}_{2}\left(n_{2}\right)-$ $\hat{\phi}_{1}\left(n_{1}\right) \hat{\phi}_{2}\left(n_{2}\right)$, we can write this profit function as (arguments omitted)

$$
\pi_{i}^{d}=d_{i} \phi_{i}-D_{12}\left(\hat{\phi}_{i} \hat{\phi}_{j}+\Delta_{\phi_{i}}\right)
$$

Here, $\hat{\phi}_{i} \hat{\phi}_{j}$ is a measure of wasted (or duplicated) advertising. It is the probability that a given overlapping viewer is informed twice, i.e., once on each outlet. This term is adjusted by $\Delta_{\phi_{i}}$ to account for viewer heterogeneity, that is, overlapping viewers become informed with a lower probability than exclusive ones. The right-hand side of (7) can then be written as

$$
\frac{\eta_{\hat{\phi}_{i} \hat{\phi}_{j}+\Delta_{\phi_{i}}}}{\eta_{\phi_{i}}}
$$

\footnotetext{
${ }^{22}$ Recall that $d_{i}\left(n_{i}\right)=D_{i}\left(n_{i}\right)+D_{12}\left(n_{1}, n_{2}\right)$.
} 
with

$$
\eta_{\hat{\phi}_{i} \hat{\phi}_{j}+\Delta_{\phi_{i}}}:=\frac{\partial\left(\hat{\phi}_{i} \hat{\phi}_{j}+\Delta_{\phi_{i}}\right)}{\partial n_{i}} \frac{n_{i}}{\hat{\phi}_{i} \hat{\phi}_{j}+\Delta_{\phi_{i}}} .
$$

Loosely speaking, the numerator of (8) is a measure of the elasticity of duplication. It tells which fraction of advertising messages gets wasted due to duplication across outlets following a one percentage point increase in the amount of messages sent. We will come back to the determinants of this term in Section 6.

This puts us in the position to provide a clear interpretation of (7). If the elasticity of overlapping viewers is large relative to the one of exclusive viewers (i.e., the left-hand side is large), the businesssharing effect prevails and advertising levels increase with entry. By contrast, if the elasticity of wasted impressions is large relative to the advertising elasticity (i.e., the right-hand side is large), the duplication effect dominates and advertising levels fall with entry.

We note that a similar intuition holds if we start from any number of incumbent outlets, not just a monopoly outlet. For example, if there are two incumbent outlets and a third one enters, there will be viewers who formerly were exclusives of one of the incumbent outlets but will now be shared with the entrant. In addition, some of the formerly overlapping viewers will now join all outlets. For both viewer types, a duplication and a business-sharing effect exists. Therefore, whether advertising levels fall or rise depends on the strength of these effects and the size of each viewer group. ${ }^{23}$

An important merit of (7) is that it spells out the effect of applying competitive pressure in terms of empirical objects. However, its insight is limited without a theory that suggests when the condition should have a particular sign. We address this issue in the next two sections. The condition asks if there are any systematic differences between the two pools of viewers that could tilt the trade-off one way or the other. The two sides of the inequality stress two different sources of dissimilarities between exclusive and overlapping viewers, both of which play a crucial role in duopoly. The left-hand side focuses on relative preferences, expressed by demand elasticities. The right-hand side focuses on potential differences in the advertising technology, expressed by elasticities of the advertising function. Given that these are two very different mechanisms, we tackle them separately. In Section 5, we add structure on the advertising functions in a way that guarantees that the right-hand side of (7) equals one. This shuts down the technological source. Results are then purely driven by systematic differences in preferences across types. In Section 6, we carry out the mirror exercise. We shut down the preferences source by using the insights gained in Section 5. As we shall see, it is possible to add structure to the joint distribution in a way that guarantees a left-hand side of $(7)$ equal to one for all $\left(n_{1}, n_{2}\right)$. Our findings in Section 6 will therefore hinge solely on technological factors. This break-up is implemented for illustrative purposes only. In principle, we could carry out the two exercises simultaneously.

\section{$5 \quad$ Viewer Preference Correlation}

To isolate how relative preferences shape the effect of competition, in this section we assume that $\hat{\phi}_{i}\left(n_{i}\right)=$ $\phi_{i}\left(n_{i}\right)$ for $i=1,2$. This amounts to considering the case where overlapping and exclusive viewers get

\footnotetext{
${ }^{23}$ See Appendix 11.2 for a formal analysis.
} 
informed with the same probability (on a single outlet). For example, this assumption is satisfied in the case when advertising technology takes the widely used exponential form $\phi_{i}(n)=\phi_{j}(n)=1-e^{-n}$ and $\phi_{12}\left(n_{1}, n_{2}\right)=\phi_{i}\left(n_{1}+n_{2}\right)=1-e^{-\left(n_{1}+n_{2}\right)} .{ }^{24}$ Using $\hat{\phi}_{i}\left(n_{i}\right)=\phi_{i}\left(n_{i}\right)$, one can easily verify that the right hand side of $(7)$ equals 1 for all $\left(n_{1}, n_{2}\right)$. So condition (7) simplifies to:

$$
\frac{\eta_{D_{12}}}{\eta_{d_{i}}}>(<) 1
$$

We now seek to identify features of the joint distribution of preferences that could lead to systematic differences in the relative elasticities of demand. A striking feature of (9) is that the effect of competition depends on the joint distribution of preferences through $\eta_{D_{12}}$ only. So any change in the joint distribution that results in a decrease of $\eta_{D_{12}}$ (for equal marginal distributions) yields downward competitive pressure on advertising levels. To bring out this effect, we add structure to the preferences. Specifically, we assume that $\left(q_{1}, q_{2}\right)$ is drawn from a bivariate normal distribution with mean $(0,0)$ and variance-covariance matrix

$$
\Sigma=\left[\begin{array}{ll}
1 & \rho \\
\rho & 1
\end{array}\right] .
$$

The parameter $\rho$ is the coefficient of correlation between $q_{1}$ and $q_{2}$ and therefore captures content 'likeness.'

We can now determine how a change in the correlation coefficient affects viewer composition.

Lemma $1 D_{12}$ is strictly increasing in $\rho$.

The lemma shows that the viewer composition changes monotonically with the correlation coefficient, i.e., a higher correlation coefficient, ceteris paribus, is equivalent to an increase in the extent of viewer overlap. In other words, an increase in correlation changes the demand composition, whereby a higher fraction of outlet $i$ 's total demand is comprised of overlapping viewers. Recall that the total demand of outlet $i$ depends only on the marginal distribution, which is unchanged by an increase in $\rho$. Therefore, only the composition is affected by $\rho$.

The result provides a key observation for the following analysis. To the best of our knowledge, it is not part of the basic collection of results on multivariate normals. It is nevertheless very useful to analyze how changes in the demand composition affect equilibrium outcomes. We also note that it holds for any combination of advertising levels $n_{1}$ and $n_{2}$.

At first thought, Lemma 1 suggests a negative relationship between $\rho$ and the equilibrium advertising level. The higher the number of overlapping viewers, the stronger the duplication effect. However, this argument is not conclusive. The reason is that a larger $\rho$ could enhance the business-sharing effect as well. Indeed, a larger $D_{12}$ leads to a larger fraction of the variation coming from overlapping viewers. Other things held constant, this suggests a positive relationship. The resulting indeterminacy is reflected by the fact that what matters is how the elasticity of the demand $D_{12}$ changes with correlation. The next lemma proves that a systematic relationship between $\eta_{D_{12}}$ and joint preferences as captured by $\rho$

\footnotetext{
${ }^{24}$ This functional form was firstly introduced in a seminal paper by Butters (1977) and has been widely used since then in applied work on advertising. It can be derived from natural primitive assumptions on the stochastic process that governs the allocation of messages to consumers.
} 
exists for symmetric advertising levels.

Lemma $2 \eta_{D_{12}}$ decreases with $\rho$ for all $n_{1}=n_{2}>0$.

Since $\eta_{d_{i}}$ does not change with $\rho$, it follows that $\eta_{D_{12}} / \eta_{d_{i}}$ decreases with $\rho$. Therefore, the lemma establishes that the set of marginal viewers is composed of relatively more exclusive viewers when correlation is higher. Figure 1 provides a geometric intuition by showing how changes in $\rho$ affect the relevant demand for a discrete increase in $n_{1}$ given $n_{2}$. In Figure 1 , outlets set $n_{1}=n_{2}=0.5$ at the outset. Suppose that outlet 1 raises its advertising level to $n_{1}=1$. At the outset, the measure of the square $A+B$ corresponds to the mass of overlapping viewers, whereas $C+D$ corresponds to the mass of exclusive viewers. Instead, when $n_{1}=1$, the measure of the square $B$ corresponds to the mass of overlapping viewers, whereas $D$ corresponds to the mass of exclusive viewers. Therefore, when outlet 1 increases its advertising level, its share of exclusive viewers goes from $(C+D) /(A+B)$ to $D / B$. Consider first $\rho=0.9$. It is evident from the right-hand side of the figure that the share of exclusives after the increase is much smaller than before. Therefore, after the increase in $n_{1}$, the demand of outlet 1 consists only of a small portion of exclusive viewers. In other words, following an increase in the advertising level, an outlet's demand composition is tilted towards overlapping viewers if the viewer preference correlation is positive. This implies that, as $n_{1}$ increases, outlet 1 loses the valuable exclusive viewers at a higher rate. Therefore, the business-sharing effect is small and is dominated by the duplication effect, resulting in downward pressure on the advertising levels. By contrast, for $\rho=0$, the composition of marginal viewers is much more balanced. The ratio $D / B$ is similar to $(C+D) /(A+B)$. Indeed, as Proposition 2 will show, for $\rho=0$ the two effects exactly offset each other. ${ }^{25}$
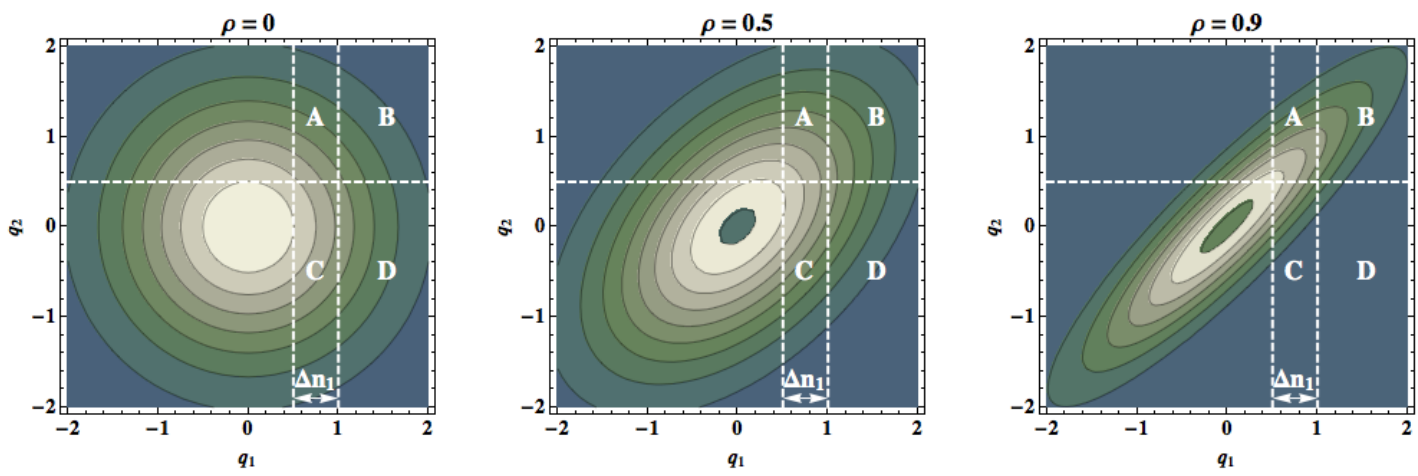

Figure 1: Contour map of $h$. Dashed lines correspond to $n_{1}=n_{2}=0.5$ and $\Delta n_{1}=0.5$.

Applying Lemma 2, we can establish the following result:

Proposition 2 An incumbent monopolist's advertising level increases upon entry of a competitor if and only if $\rho$ is negative. That is,

$$
\operatorname{sign}\left(n_{i}^{d}-n_{i}^{m}\right)=\operatorname{sign}\left(\eta_{D_{12}}-\eta_{d_{i}}\right)=-\operatorname{sign}(\rho)
$$

\footnotetext{
${ }^{25}$ We restrict our attention to a bivariate normal distribution. However, we conjecture that the result of Lemma 2 holds more broadly. The reason is that the result depends on the fact that the bivariate normal distribution satisfies a generalization of the familiar Monotone Hazard Rate Condition to two-dimensional variables. (We show this in the proof of Lemma 2.) We conjecture that the same result applies to other distributions which satisfy this condition.
} 
where all functions are evaluated at $n_{i}=n_{i}^{m}$ and $n_{j}=n_{j}^{d}$.

A positive correlation coefficient leads to a fall in advertising levels with competition, whereas a negative one leads to higher advertising levels. Before moving on, we use this result in two different ways. First, we discuss the implied strategic considerations of outlets when choosing which kind of content to produce. Second, we discuss how this result can be used as a first empirical test of the theory.

\section{Implications for Content Choice}

While content has been kept exogenous so far, a natural application of this model is content choice. In particular, it allows us to add to the ongoing debate on "competition and diversity" in the media, which is often spelled out as "ideological" diversity. The exercise relies on two premises: 1) potential entrants can affect the degree of correlation at a 'content-production' stage. This stage is akin to product positioning in standard models of product differentiation; 2) a decrease in $\rho$ can be read as an increase in the supply of more diverse content. Our aim is not to provide a full-fledged model of differentiation, which would be largely outside the scope of the paper. Rather, we seek here to identify broad mechanisms that we do not expect to be sensitive to a particular model specification: 1) Would an entrant that caters to the same viewers as the incumbent be more or less profitable than an outlet that caters to those who find the incumbent unappealing? 2) In light of Proposition 2, do strategic considerations enhance or reduce the incentives to differentiate one's content from the rival's?

What we have in mind is a simple two-stage game. At stage 1, an entrant observes the content of the incumbent and chooses the extent of differentiation $-\rho$ so as to maximize its profits minus an investment cost that possibly depends itself on $\rho{ }^{26}$ At stage 2, competition takes place as described in Section 2 . Given a well-behaved problem, in equilibrium the marginal benefit of differentiation

$$
\frac{d \pi_{i}^{d}}{d-\rho}=\left(\frac{\partial D_{12}}{\partial \rho}-\frac{\partial n_{j}}{\partial \rho}\left(\frac{\partial\left(\phi_{12}-\hat{\phi}_{j}\right)}{\partial n_{j}} \frac{1}{\phi_{i}+\hat{\phi}_{j}-\phi_{12}}-\frac{\partial D_{12}}{\partial n_{j}}\right)\right)\left(\phi_{i}+\hat{\phi}_{j}-\phi_{12}\right),
$$

is equated to the marginal cost. The key observation here is that this marginal benefit is not necessarily monotonically decreasing in $\rho$, so that $\rho>0$ can indeed hold in equilibrium as a result of firms trying to 'soften' competition, much as in standard models of product differentiation. To build intuition suppose that the entrant is considering supplying similar content (i.e. $\rho>0$ ). We can then evaluate the marginal benefit and consider its sign which shapes the incentives to differentiate. The first term is positive. It captures the basic insight that decreasing correlation leads to capturing relatively more exclusive viewers, which are more valuable to advertisers. So other things held constant, the entrant has an incentive to invest in 'diverse' content (or diminish $\rho$ ). The second term in parenthesis accounts for the rival's reaction. As discussed, a lower $\rho$ results in an equilibrium in which the rival outlet competes more aggressively for advertising dollars by increasing its supply of ads. This mechanism, which we conventionally refer to as the 'strategic' one, may point in the opposite direction, as more ads from the rival reduce the extent of rent extraction from overlapping viewers. In general, the overall effect is ambiguous and which one prevails is ultimately an empirical question.

Interestingly, our 'direct' and 'strategic' effect can have opposite forces than in standard models of

\footnotetext{
${ }^{26}$ For instance $c(1-\rho)$ with $c^{\prime}>0$ and $c^{\prime \prime}>0$ would capture the idea that duplication $\rho=1$ is almost costless while differentiation is increasingly costly.
} 
differentiation (see, for example, d'Aspremont, Gabszewicz and Thisse (1979) on horizontal differentiation or Shaked and Sutton (1982) on vertical differentiation). In these models, the strategic effect is that firms become more differentiated to 'soften' competition while the direct effect is that firms have a smaller secured demand (a smaller 'hinterland') which lowers profits. In our case, the opposite could hold, escaping competition through less differentiation.

Note that these questions have not been addressed in previous works, as these models rely either on Hotelling spatial models or assume a representative viewer. In the first case, the correlation between viewers' preferences is assumed to be perfectly negative, i.e., the viewer who likes outlet $i$ most likes outlet $j$ least, while in the second case viewers are homogeneous by assumption.

\section{Empirical Analysis}

As a reality check, Appendix 11.3 contains a first pass to test Proposition 2. We conduct an empirical exercise using data on the U.S. Broadcasting TV industry. The exercise exploits variation in the extent of competitive pressure brought about by entry and exit of TV channels in the Basic Cable lineup in the $80 \mathrm{~s}$ and $90 \mathrm{~s}$. The empirical strategy is to regress strategic choices, which we observe, (here the logarithm of the average number of advertising slots per hour supplied by the networks) on a measure of entry and a number of controls. We find that a decrease in preference correlation is associated with an increase in the advertising levels as predicted (and vice versa). Furthermore, this evidence is in line with the anecdotal evidence on the positive impact of the entry of FOX News on the advertising level supplied by MSNBC or CNN, which is often referred to as the "Fox News Puzzle".

There are mainly two difficulties with the analysis, which is why we decided to relegate it to the appendix. A first one is the issue of entry endogeneity on incumbent performance. In general, it is hard to instrument for entry (see e.g., Berry and Reiss, 2007) and we do not have exploitable variation for this purpose. A second one is that we do not directly observe consumers' preferences and thus their correlation across different outlets. This said, we believe it is reasonable to assume that those who watch ESPN are more likely to watch ESPN2 or FoxSports. So we make assumptions on how the content of the entrants in a particular segment relates to that of the incumbents.

\section{Advertising Technology}

As outlined above, competition comes hand-in-hand with duplication. In duopoly, multi-homers receive the same ads from two different sources. We now explore if this fact together with diminishing marginal returns injects downward pressure on advertising levels. To focus on the advertising technology, we shut down preference sources and assume throughout this section that $\eta_{D_{12}}=\eta_{d_{i}}$. As shown in the last section, this assumption holds, for example, if the valuations for the two outlets are standard normally distributed and independent of each other (i.e., $\rho=0$ ). Condition (7) tells us that in this particular case competition reduces advertising levels if and only if

$$
1<\frac{\eta_{\hat{\phi}_{i} \hat{\phi}_{j}+\Delta_{\phi_{i}}}}{\eta_{\phi_{i}}}
$$

If the above condition is violated, the advertising level of an outlet in duopoly is higher than in monopoly, despite overlapping viewers redundantly being reached multiple times. (Precisely, overlapping viewers get at least one message from both sources with probability $\left.\hat{\phi}_{i} \hat{\phi}_{j}\right)$. The next proposition shows that 
competition is shaped by the outlet's relative elasticities of informing different kinds of viewers.

Proposition 3 The comparison between advertising levels in monopoly and in duopoly depends solely on the relative elasticity of the reach of exclusive versus overlapping viewers. In particular, an incumbent monopolist's advertising level increases upon entry of a competitor if and only if

$$
\operatorname{sign}\left(n_{i}^{d}-n_{i}^{m}\right)=\operatorname{sign}\left(\eta_{\hat{\phi}_{i}}-\eta_{\phi_{i}}\right)
$$

where all functions are evaluated at $n_{i}=n_{i}^{m}$ and $n_{j}=n_{j}^{d}$.

The proposition shows that advertising increases with entry if the elasticity to inform an overlapping viewer on a single outlet is higher than the elasticity to inform an exclusive viewer. To grasp the intuition for this result, recall that the first elasticity is defined as $\left(\partial \hat{\phi}_{i} / \partial n_{i}\right) n_{i} / \hat{\phi}_{i}$, whereas the second is defined as $\left(\partial \phi_{i} / \partial n_{i}\right) n_{i} / \phi_{i}$. The duplication effect is captured by the respective first terms of the elasticities, $\partial \hat{\phi}_{i} / \partial n_{i}$ and $\partial \phi_{i} / \partial n_{i}$. Here, $\partial \phi_{i} / \partial n_{i}$ is (weakly) larger than $\partial \hat{\phi}_{i} / \partial n_{i}$, that is, advertising to exclusive viewers is more profitable. Everything else constant, this induces the monopolist to set a higher advertising level. However, it is not the derivative alone that matters for the equilibrium outcome but the elasticity. In particular, overlapping viewers are harder to reach than exclusive viewers for a given number of ads, that is, $\hat{\phi}_{i} \leq \phi_{i}$. This implies that the second term of $\eta_{\hat{\phi}_{i}}$ is larger than the one of $\eta_{\phi_{i}}$, which induces a countervailing force. Intuitively, the probability that a multi-homing viewer is informed is low and so an additional ad to this viewer might be worth more than an ad to an exclusive viewer. Therefore, the overall effect is ambiguous.

To gain further insights, consider the technology of the exponential form with the feature that overlapping viewers become informed with a lower probability than exclusives, that is, $\phi_{i}(n)=1-e^{-b n}$ and $\hat{\phi}_{i}(n)=1-e^{-\hat{b} n}$, with $b \geq \hat{b}$. It is easy to show that (10) then simplifies to

$$
\operatorname{sign}\left(n_{i}^{d}-n_{i}^{m}\right)=\operatorname{sign}\left(\eta_{\hat{\phi}_{i}}-\eta_{\phi_{i}}\right)=\operatorname{sign}\left((b-\hat{b}) e^{-(b+\hat{b}) n_{i}^{m}}+\hat{b} e^{-\hat{b} n_{i}^{m}}-b e^{-b n_{i}^{m}}\right) .
$$

It is readily verified that the sign of (11) is strictly larger than zero for all $0<\hat{b}<b$ and is exactly zero for $\hat{b}=0$ and $\hat{b}=b$. Interestingly, this shows that for all interior values of $\hat{b}$, advertising levels in duopoly are larger than in monopoly. The example demonstrates that the countervailing force identified above in conjunction with the business-sharing effect dominates the duplication effect. The exception are the cases in which $\hat{b}=0$ and $\hat{b}=b$. In the latter case, reaching exclusive and overlapping viewers (on a single outlet) is equally effective, leading to the same trade-off in monopoly and duopoly. In the other extreme case, $\hat{b}=0$, overlapping viewers are of zero value. Therefore, an outlet in duopoly only cares about its exclusive viewers when choosing the advertising level. Since a monopolistic outlet has only exclusive viewers, the trade-off and the equilibrium advertising level in both scenarios are again equivalent.

\section{Outlet Mergers}

An important question in media economics is the effect of outlet mergers on market outcomes. To address this question, in this section we contrast the duopoly outcome with the outcome that a hypothetical monopolist who controls both outlets would implement. We obtain the following result:

Proposition 4: The equilibrium advertising level in duopoly is strictly lower than under joint own- 
ership (i.e., $n_{i}^{d}<n_{i}^{j o}$ ) if $\hat{\phi}_{j}<\phi_{j}$ and the two advertising levels are equivalent if $\hat{\phi}_{j}=\phi_{j}$.

To build intuition, consider first the case $\hat{\phi}_{i}=\phi_{i}$. When marginally increasing $n_{i}$, a monopolistic owner controlling both outlets loses some multi-homing viewers who become single-homing viewers on outlet $j$. With the first kind of viewers the monopolist loses $\phi_{12}$, while with the second he gains $\phi_{j}$. In duopoly, when an outlet increases $n_{i}$, it loses some multi-homing viewers who are worth $\phi_{12}-\phi_{j}$. But this implies that the trade-offs in both market structures are the same. ${ }^{27}$ As a consequence, we obtain that the ownership structure has no effect on advertising levels.

If instead $\hat{\phi}_{i}<\phi_{i}$, overlapping viewers can be reached with a lower probability by advertisers than single-homing viewers. Therefore, competing outlets can then extract $\left(\phi_{12}-\hat{\phi}_{j}\right)>\left(\phi_{12}-\phi_{j}\right)$ from advertisers. This implies that when losing overlapping viewers, an outlet in duopoly loses more than a joint owner does. Therefore, the business-sharing effect for a joint owner is larger than for competing outlets, leading to lower advertising levels in duopoly. This provides a countervailing force to the standard intuition that monopolists keep the advertising levels low to extract higher transfers from advertisers. In our model, the business-sharing effect can lead to the opposite conclusion.

To gain further intuition, let us explain the result that $n_{i}^{d}=n_{i}^{j o}$ for $\hat{\phi}_{j}=\phi_{j}$ in more detail. The following decomposition of $\Pi_{i}^{d}$ may aid intuition for the equivalence result. In particular, $\Pi_{i}^{d}$ can be written as

$$
\Pi_{i}^{d}=\Pi^{j o}-\phi_{j}\left(D_{j}-D_{12}\right)
$$

The profit of a duopolist is equivalent to the aggregate profit minus a correction term which only depends on the demand and advertising technology of the rival outlet. This is reminiscent of the payoff induced by Clarke-Groves mechanisms (Clarke, 1971; Groves, 1973). Each agent's payoff is equal to the entire surplus minus a constant term - since the sum of $D_{j}$ and $D_{12}$ is unaffected by outlet $i$ 's choices in either or both competition - which is equal to the payoff that the other agents would get in his absence. ClarkeGroves mechanisms implement socially efficient choices, here represented by the joint monopoly solution. If $\hat{\phi}_{j} \neq \phi_{j}$, this result no longer holds.

The result that advertising levels do not depend on the ownership structure for $\hat{\phi}_{j}=\phi_{j}$ is also reminiscent of common agency models (e.g., Bernheim and Whinston, 1986), that predict equivalent allocations when firms compete and when they cooperate. However, common agency models feature a single agent who contracts with multiple principals instead of a continuum of agents, as in our framework. In particular, if there is only a single advertiser - or, equivalently, if all advertisers can coordinate their choices $^{28}$ — even in models featuring single-homing of viewers, the equilibrium advertising levels are the same under duopoly and joint ownership. ${ }^{29}$ In contrast to this, advertisers cannot coordinate their choices

\footnotetext{
${ }^{27}$ Note that in both cases increasing $n_{i}$ also implies losing some single-homing viewers on outlet $i$. But the loss from these viewers is exactly the same for the monopolist and the duopolist.

${ }^{28}$ For an analysis of consumer coordination in outlet competition in a setting with positive network externalities, see Ambrus and Argenziano (2009).

${ }^{29}$ To see this consider the case in which viewers join either outlet $i$ or $j$, implying that $D_{12}=0$. If there is only a single advertiser, the transfer that outlet $i$ can charge to make the advertiser accept is the incremental value of the outlet, i.e., $u\left(n_{1}^{d}, n_{2}^{d}\right)-u\left(0, n_{j}^{d}\right)$. In the either/or framework, $u\left(n_{1}^{d}, n_{2}^{d}\right)=D_{1}\left(n_{1}^{d}, n_{2}^{d}\right) \phi_{1}\left(n_{1}\right)+D_{2}\left(n_{1}^{d}, n_{2}^{d}\right) \phi_{2}\left(n_{2}\right)$, while $u\left(0, n_{j}^{d}\right)=D_{j}\left(0, n_{j}^{d}\right) \phi_{j}\left(n_{j}\right)$. Hence,

$$
\Pi_{i}^{d}=D_{1}\left(n_{1}^{d}, n_{2}^{d}\right) \phi_{1}\left(n_{1}\right)+D_{2}\left(n_{1}^{d}, n_{2}^{d}\right) \phi_{2}\left(n_{2}\right)-D_{j}\left(0, n_{j}^{d}\right) \phi_{j}\left(n_{j}\right)
$$
}


in our model. Therefore, the mechanism described above, which leads to the equivalence result in our model, is distinct to the one in common agency frameworks.

\section{Is There too much Advertising?}

A common theme in media markets is that the market provides an inefficiently high quantity of advertising. To address this, we proceed by characterizing the socially optimal allocation. As mentioned, $q_{i}-\gamma n_{i}$ is the utility of a single-homing viewer of outlet $i$ and $q_{1}-\gamma n_{1}+q_{2}-\gamma n_{2}$ is the utility of a multi-homing viewer. Social welfare is given by

$$
\begin{aligned}
W & =\int_{\gamma n_{1}}^{\infty} \int_{0}^{\gamma n_{2}}\left(q_{1}-\gamma n_{1}\right) h\left(q_{1}, q_{2}\right) d q_{2} d q_{1}+\int_{0}^{\gamma n_{1}} \int_{\gamma n_{2}}^{\infty}\left(q_{2}-\gamma n_{2}\right) h\left(q_{1}, q_{2}\right) d q_{2} d q_{1} \\
& +\int_{\gamma n_{1}}^{\infty} \int_{\gamma n_{2}}^{\infty}\left(q_{1}-\gamma n_{1}+q_{2}-\gamma n_{2}\right) h\left(q_{1}, q_{2}\right) d q_{2} d q_{1}+D_{1} \phi_{1}+D_{2} \phi_{2}+D_{12} \phi_{12} .
\end{aligned}
$$

Comparing the equilibrium advertising level with the socially efficient advertising level we obtain the following result:

Proposition 5: Equilibrium advertising levels are inefficiently high if $\hat{\phi}_{i}$ is close to $\phi_{i}$.

To grasp the intuition behind the result, it is useful to consider the incentives of the joint monopoly. Under our assumptions the monopolist fully internalizes advertisers' welfare. On the contrary, it does not internalize viewers' welfare. More precisely, it only cares about viewers' utilities inasmuch as they contribute to advertising revenue, while the nuisance costs from advertising are not taken into account. This leads to inefficiently high advertising levels. From our last section, we know that competing outlets implement the same advertising levels as long as $\hat{\phi}_{j}=\phi_{j}$. Therefore, the equilibrium allocation in duopoly leads to an advertising level which is too large from a social perspective.

The condition in Proposition 5 is sufficient, but not necessary. In particular, the result also holds when $\hat{\phi}_{i}$ is much lower than $\phi_{i}$ as long as viewer disutility from ads is sufficiently high. The reason is again that outlets do not take viewers' utilities directly into account in their advertising choice. The result can only be reversed if the disutility from ads is small and $\hat{\phi}_{i}<<\phi_{i}$ because, as we know from the last section advertising levels in duopoly are then much lower than with joint ownership.

Proposition 5 should be interpreted with caution. The overprovision result hinges on the assumption that advertisers are homogeneous. If advertisers are heterogeneous with respect to their product valuations, an extensive margin comes into play in addition to the intensive margin considered so far. This extensive margin arises because, as in previous literature, a outlet owner trades off the marginal profit from an additional advertiser with the profits from inframarginal advertisers. This effect coupled with our result can either lead to socially excessive or socially insufficient advertising levels.

We note that our conclusions differ from those obtained in previous models. For instance, in Anderson and Coate (2005) competition for viewers always reduces advertising levels relative to monopoly, which can lead to inefficiently low advertising levels, even with homogeneous advertisers. In addition, the

The first two terms are equivalent to a monopolist's profit, while the last term is independent of $n_{i}^{d}$. Therefore, the first-order conditions for monopoly and duopoly coincide. 
redistributive impact of a merger is very different in both models. In our model, a joint owner can fully expropriate advertisers, whereas competing outlets cannot, implying that advertisers are hurt by a merger. In contrast, in Anderson and Coate (2005) a merger leads to an increase in the advertising level and a lower advertising price. Hence, advertisers are better off after a merger.

\section{$9 \quad$ Heterogeneous Advertisers}

In this section, we discuss how the trade-off characterized in Proposition 1 extends to advertisers with heterogeneous product values $\omega$, as in Anderson and Coate (2005). As we will show, the key insights obtained in the analysis with homogeneous advertisers carries through to heterogeneous advertisers. In particular, outlet competition is also characterized by the tension between the duplication and businesssharing effect. This holds although the analysis is more involved compared to homogeneous advertisers, as we need to characterize an entire contract schedule (i.e., the optimal screening contracts) offered by outlets $\left(m_{i}(\omega), t_{i}(\omega)\right)$, instead of only a single transfer-quantity pair $\left(m_{i}, t_{i}\right) .{ }^{30}$

Consider the following extension of our baseline model. The value of informing a viewer, $\omega$, is distributed according to a smooth c.d.f. $F$ with support $[\underline{\omega}, \bar{\omega}], 0<\underline{\omega} \leq \bar{\omega}$, that satisfies the monotone hazard rate property. The value $\omega$ is private information to each advertiser. The timing of the game is the same as before. In the first stage, each outlet $i$ announces its total advertising level $n_{i}$. Afterwards, consumers decide which outlet to join. Given these decisions, each outlet offers a menu of contracts consisting of a transfer schedule $t_{i}:=[0, \bar{m}] \rightarrow \mathbb{R}$ defined over a compact set of advertising levels. $t_{i}(m)$ is the transfer an advertiser has to pay to get an advertising intensity $m$ from outlet $i$. In the final stage, as before, advertisers decide which outlet to join. In what follows, we define $n=\left(n_{1}, n_{2}\right)$.

Let us start with the monopoly case. With an abuse of notation we still use $\omega u\left(m_{i}, n_{i}\right)$ to denote the surplus of advertiser type $\omega$ from advertising intensity $m_{i}$. The overall utility of an advertiser depends on the transfer schedule in addition to the surplus. If $m_{i}(\omega)$ denotes the optimal intensity chosen by type $\omega$, then outlets $i$ 's problem in case of monopoly is

$$
\Pi=\max _{t_{i}(\cdot)} \int_{\underline{\omega}}^{\bar{\omega}} t_{i}\left(m_{i}(\omega)\right) d F(\omega) .
$$

By choosing the optimal menu of contracts, the monopolist determines which advertiser types to exclude, that is, $m_{i}(\omega)=0$ for these types, and which advertiser types will buy a positive intensity. We denote the marginal advertiser by $\omega_{0}^{m}$. Problem (12) can be expressed as a standard screening problem:

$$
\begin{array}{cl} 
& \Pi=\max _{\omega_{0}^{m}, m_{i}(\omega)} \int_{\omega_{0}^{m}}^{\bar{\omega}} t_{i}\left(m_{i}(\omega)\right) d F(\omega) \\
\text { subject to } & m_{i}(\omega)=\arg \max _{m_{i}} v_{i}^{m}\left(m_{i}, \omega, n_{i}\right)-t_{i}\left(m_{i}\right), \\
& v_{i}^{m}\left(m_{i}(\omega), \omega, n_{i}\right)-t_{i}\left(m_{i}(\omega)\right) \geq 0 \text { for all } \omega \geq \omega_{0}^{m}, \\
& \int_{\omega_{0}^{m}}^{\bar{\omega}} m_{i}(\omega) d F(\omega) \leq n_{i},
\end{array}
$$

\footnotetext{
${ }^{30}$ Our results also hold when outlets can perfectly discriminate between advertisers. In that case, the results for each type are the same as the ones in case of homogeneous advertisers.
} 
where $v_{i}^{m}\left(m_{i}, \omega, n_{i}\right):=\omega d_{i}\left(n_{i}\right) \phi_{i}\left(m_{i}\right)$ denotes the net value of advertising intensity $m_{i}$ to type $\omega$ in the monopoly case. The first constraint is the incentive-compatibility constraint and the second one the participation constraint. The third one is the capacity constraint specifying that the aggregate advertising level cannot exceed the one specified by the outlet in the first stage. Provided that the function $v_{i}^{m}\left(m_{i}, \omega, n_{i}\right)$ satisfies the standard regularity conditions in the screening literature, we can apply the canonical screening methodology Our assumptions on the viewer demand $d_{i}\left(n_{i}\right)$ and on the advertising technology $\phi_{i}\left(m_{i}\right)$ ensure that $v_{i}^{m}$ is continuous and increasing in $\omega$. It also has strictly increasing differences in $(m, \omega)$.

Evidently, the capacity constraint will be binding at the optimal solution since it can never be optimal for the monopolist to announce a strictly larger advertising level than the one it uses. Applying the above-mentioned methodology, we can transform the maximization problem to get

$$
\Pi=\max _{\omega_{0}^{m}, m_{i}(\omega)} \int_{\omega_{0}^{m}}^{\bar{\omega}}\left(\omega-\frac{1-F(\omega)}{f(\omega)}\right) d_{i}\left(n_{i}\right) \phi_{i}\left(m_{i}(\omega)\right) d F(\omega)
$$

subject to $n_{i}=\int_{\omega_{0}^{m}}^{\bar{\omega}} m_{i}(\omega) d F(\omega)$.

We show in Appendix 11.2 that the optimal advertising level $n_{i}$ can be characterized by the following equation:

$$
\int_{\omega_{0}^{m}}^{\bar{\omega}}\left(\omega-\frac{1-F(\omega)}{f(\omega)}\right)\left(\tilde{d}_{i} \frac{\partial \phi_{i}}{\partial m_{i}}+\frac{\partial d_{i}}{\partial n_{i}} \phi_{i}\right) d F(\omega)=0
$$

with $\tilde{d}_{i}:=\left(1-F\left(\omega_{0}^{m}\right)\right) d_{i}$. We can compare this characterization with the one for homogeneous advertisers given by (5). Due to the information rent that is required for incentive compatibility, the outlet can no longer extract the full rent from advertisers but only a fraction of it. This is expressed by the first bracket in the integral. Inspecting the second bracket, the expression is analogous to the one with homogeneous advertisers. Note that in the latter case $m_{i}=n_{i}$ implies that the derivative was taken with respect to $n_{i}$ in both terms. The above expression instead accounts for the fact that the optimal allocation $m_{i}(\omega)$ is heterogeneous across types. A second difference comes from the first term in the second bracket where we have $\tilde{d}_{i}$ instead of $d_{i}$. When changing $m_{i}$, only those advertisers who participate are affected. This is only a mass of $1-F\left(\omega_{0}^{m}\right)$. By contrast, with homogeneous advertisers all of them are active in equilibrium.

Therefore, with heterogeneous advertisers the equation characterizing $n_{i}$ trades off the cost and benefits of increasing $n_{i}$ over the whole mass of participating advertisers, implying that the average costs and benefits are important. However, the basic trade-off for homogeneous advertisers and heterogeneous advertisers is the same. In particular, the first term in the second bracket represents the average marginal profit from increased reach on infra-marginal consumers, whereas the second term represents the average loss from marginal consumers who switch off.

Let us now turn to the optimal advertising levels in duopoly. The goal is to characterize the bestreply tariff $t_{i}\left(m_{i}\right)$ given outlet $j$ 's choice $t_{j}\left(m_{j}\right)$. As in the monopoly case, it is possible to rewrite this problem as a standard screening problem. To this end, denote by $\omega u\left(m_{1}, m_{2}, n\right)$ the surplus of type $\omega$ from advertising intensities $\left(m_{1}, m_{2}\right)$. If $m_{i}(\omega)$ denotes the optimal quantity chosen by type $\omega$, then outlets $i$ 's optimization problem is

$$
\Pi=\max _{\omega_{0}^{i}, m_{i}(\omega)} \int_{\omega_{0}^{i}}^{\bar{\omega}} t_{i}\left(m_{i}(\omega)\right) d F(\omega)
$$




$$
\begin{array}{ll}
\text { subject to } & m_{i}(\omega)=\arg \max _{m_{i}} v_{i}^{d}\left(m_{i}, \omega, n\right)-t_{i}\left(m_{i}\right), \\
& v_{i}^{d}\left(m_{i}(\omega), \omega, n\right)-t_{i}\left(m_{i}(\omega)\right) \geq 0 \text { for all } \omega \geq \omega_{0}^{i}, \\
& \int_{\omega_{0}^{i}}^{\bar{\omega}} m_{i}(\omega) d F(\omega) \leq n_{i},
\end{array}
$$

where $v_{i}^{d}\left(m_{i}, \omega, n\right):=\max _{y} \omega u\left(m_{i}, y, n\right)-t_{j}(y)-\max _{y^{\prime}}\left(\omega u\left(0, y^{\prime}, n\right)-t_{j}\left(y^{\prime}\right)\right)$, with $u\left(m_{i}, y, n\right):=$ $D_{i}\left(n_{1}, n_{2}\right) \phi_{i}\left(m_{i}\right)+D_{j}\left(n_{1}, n_{2}\right) \phi_{j}(y)+D_{12}\left(n_{1}, n_{2}\right) \phi_{12}\left(m_{i}, y\right)$.

Note that the sole difference with respect to the monopoly case is that each advertiser's outside option accounts for the possibility of accepting the rival's offer. Hence, $v_{i}^{d}\left(m_{i}, \omega, n\right)$ is larger than $v_{i}^{m}\left(m_{i}, \omega, n_{i}\right)$. Again, our assumptions about the viewer demands $D_{i}\left(n_{1}, n_{2}\right)$ and $D_{12}\left(n_{1}, n_{2}\right)$ and about the advertising technology $\phi_{i}\left(m_{i}\right)$ and $\phi_{12}\left(m_{1}, m_{2}\right)$ ensure that $v_{i}^{d}$ is continuous and increasing in $\omega$. It also has strict increasing differences in $(m, \omega)$.

In the appendix we show by following the methodology of Martimort and Stole (2009) that it is possible to characterize the best-reply allocation as the solution to

$$
\int_{\omega_{0}^{i}}^{\bar{\omega}}\left(\omega-\frac{1-F(\omega)}{f(\omega)}\right)\left(\tilde{d}_{i} \frac{\partial \phi_{i}}{\partial m_{i}}+\frac{\partial d_{i}}{\partial n_{i}} \phi_{i}+\tilde{D}_{12} \frac{\partial\left(\phi_{12}-\phi_{i}-\hat{\phi}_{j}\right)}{\partial m_{i}}+\frac{\partial D_{12}}{\partial n_{i}}\left(\phi_{12}-\phi_{i}-\hat{\phi}_{j}\right)\right) d F(\omega)+\kappa=0,
$$

with $\tilde{d}_{i}:=\left(1-F\left(\omega_{o}^{i}\right)\right) d_{i}, \tilde{D}_{12}:=\left(1-F\left(\omega_{o}^{i}\right)\right) D_{12}$, and $\kappa$ defined in Appendix 11.2. Ignoring $\kappa$ for the moment, it is evident that this optimal duopoly solution (15) is the analog of condition (6) accounting for the business sharing and duplication effect with heterogeneous advertisers.

Let us finally turn to $\kappa$. When changing the advertising intensity of type $\omega$, outlet $i$ has to take into account that such a different intensity also affects the advertisers' demand from the rival outlet, $m_{j}$, given the posted schedule $t_{j}(\cdot)$. Intuitively, the higher the number of advertising messages on outlet $i$, the lower the utility from one additional ad on outlet $j$. This channel brings in new competitive forces that are absent with homogeneous advertisers. These forces are specific to the contracting environment considered and in addition to the ones discussed so far. To stress this, we note in the appendix that if the rival outlet were to offer a single quantity-transfer pair (or, in other words, were to implement an incentive compatible allocation flat across all active types) then $\kappa=0$ and the best-reply would still be characterized by (15).

\section{Conclusion}

This paper presented a model of outlet competition with overlapping viewerships, allowing for fairly general viewer demand and advertising technologies. We emphasize the role that viewer composition plays for market outcomes, and identify novel competitive effects, such as the duplication and the businesssharing effect.

The generality of the framework allows the model to serve as a useful building block to tackle a variety of questions. For example, we took the quality of outlets to be exogenous in our analysis. Yet, competition in media markets (and in many other industries) often works through quality. Our model can be used to investigate whether markets in which users can be active on multiple outlets lead to higher or lower quality than those in which users are primarily active on a single outlet. Another interesting question pertains to pricing tools. We considered the case in which outlets offer contracts consisting of an advertising level and a transfer, but in some industries firms primarily charge linear prices. How then do our results depend on the contracting environment? Also, do linear prices lead to a more or less 
competitive outcome? We leave these questions for future research.

Our model is also not restricted to the media markets context. In particular, a characterizing feature of our model is that consumers are multi-stop shoppers, i.e., can patronize multiple firms, but that a firm's revenue is lower for a consumer who buys from several other firms. Hence our model contributes to understanding competition in settings where firms care not only about the overall demand but also about its composition. Such settings arise naturally when serving different types of customers yield different revenues from other sources (as in our model), as well as when there are consumption externalities among customers. 


\section{Appendix}

\subsection{Proof of Propositions}

\section{Proof of Claim 1:}

First suppose that there is a non-singleton menu of contracts $\left(t_{i}^{k}, m_{i}^{k}\right)_{k=1}^{K}$ offered by outlet $i$ such that each of these contracts is accepted by some advertisers. Then advertisers have to be indifferent between these contracts. Let $F(k)$ denote the cumulative density of advertisers accepting some contract $\left(t_{i}^{k^{\prime}}, m_{i}^{k^{\prime}}\right)$ for some $k^{\prime} \leq k$. Then, by strict concavity of $\phi_{i}$ and $\phi_{12}$, if outlet $i$ instead offered a single contract $\left(F(K) E\left(t_{i}^{k}\right), F(K) E\left(m_{i}^{k}\right)\right)$, where the expectations are taken with respect to $F$, each advertiser would strictly prefer to accept the contract, resulting in the same total advertising level and profit for the outlet. But then outlet $i$ could increase profits by offering a single contract $\left(F(K) E\left(t_{i}^{k}\right)+\varepsilon, F(K) E\left(m_{i}^{k}\right)\right)$, for a small enough $\varepsilon>0$, since such a contract would still guarantee acceptance from all advertisers. The same logic can be used to establish that it cannot be in equilibrium that a single contract $\left(t_{i}, m_{i}\right)$ is offered but only a fraction of advertisers $F(1)<1$ accept it, since offering $\left(F(1) \times t_{i}+\varepsilon, F(1) \times m_{i}\right)$ for small enough $\varepsilon>0$ would guarantee acceptance by all advertisers and generate a higher profit for outlet $i$.

The above arguments establish that the total realized advertising level on outlet $i$ is $m_{i}$, the intensity specified in the single contract offered by $i$. It cannot be that $m_{i}>n_{i}$, since then by assumption the outlet's payoff would be negative. Moreover, since $\phi_{i}$ and $\phi_{12}$ are strictly increasing, it cannot be that $m_{i}<n_{i}$, since then the outlet could switch to offering a contract $\left(t_{i}+\varepsilon, n_{i}\right)$, which for small enough $\varepsilon>0$ would guarantee acceptance by all advertisers and generate a higher profit for outlet $i$. Thus $m_{i}=n_{i}$.

Finally, note that $t_{1}<u\left(n_{1}, n_{2}\right)-u\left(0, n_{2}\right)$ implies that outlet 1 could charge a higher transfer and still guarantee the acceptance of all advertisers, while $t_{1}>u\left(n_{1}, n_{2}\right)-u\left(0, n_{2}\right)$ would contradict that all advertisers accept both outlets' contracts. Hence, $t_{1}=u\left(n_{1}, n_{2}\right)-u\left(0, n_{2}\right)$. A symmetric argument establishes that $t_{2}=u\left(n_{1}, n_{2}\right)-u\left(n_{1}, 0\right)$.

The proof of Claim 2 proceeds exactly along the same lines and is therefore omitted.

\section{Proof of Proposition 1:}

We know that the equilibrium advertising level in case of duopoly is given by (6), while the equilibrium advertising level of a single outlet monopolist is given by (5). To check if advertising levels rise with entry, let us evaluate (6) at $n_{i}^{m}$ and $n_{j}^{d}$. Since the first terms in equations (5) and (6) are the same, we have $n_{i}^{d}>n_{i}^{m}$ if and only if

$$
D_{12} \frac{\partial\left(\phi_{12}-\hat{\phi}_{j}-\phi_{i}\right)}{\partial n_{i}}+\frac{\partial D_{12}}{\partial n_{i}}\left(\phi_{12}-\hat{\phi}_{j}-\phi_{i}\right)>0 .
$$

Due to the fact that the objective functions are single-peaked, it follows that the incumbent's equilibrium advertising level in duopoly is larger than the equilibrium advertising level in monopoly if the marginal profit evaluated at the pre-entry advertising level is positive, given that outlet $j$ sets $n_{j}^{d}$. Rearranging this inequality gives (acknowledging the fact that $\phi_{12}-\phi_{i}-\hat{\phi}_{j}<0$ )

$$
-\frac{\partial D_{12}}{\partial n_{i}} \frac{n_{i}}{D_{12}}>\left(\frac{\partial\left(\phi_{12}-\hat{\phi}_{j}-\phi_{i}\right)}{\partial n_{i}}\right) \frac{n_{i}}{\phi_{12}-\hat{\phi}_{j}-\phi_{i}} .
$$


We can write this inequality as

$$
-\frac{\partial D_{12}}{\partial n_{i}} \frac{n_{i}}{D_{12}}>\frac{\partial\left(\phi_{i}+\hat{\phi}_{j}-\phi_{12}\right)}{\partial n_{i}} \frac{n_{i}}{\phi_{i}+\hat{\phi}_{j}-\phi_{12}}
$$

Using our definitions

$$
\eta_{D_{12}}:=-\frac{\partial D_{12}}{\partial n_{i}} \frac{n_{i}}{D_{12}}
$$

and

$$
\eta_{\phi_{i}+\hat{\phi}_{j}-\phi_{12}}:=\frac{\partial\left(\phi_{i}+\hat{\phi}_{j}-\phi_{12}\right)}{\partial n_{i}} \frac{n_{i}}{\phi_{i}+\hat{\phi}_{j}-\phi_{12}},
$$

we can rewrite (16) as $\eta_{D_{12}}>\eta_{\phi_{i}+\hat{\phi}_{j}-\phi_{12}}$. Dividing this expression by $\eta_{d_{i}}>0$, we obtain $\eta_{D_{12}} / \eta_{d_{i}}>$ $\eta_{\phi_{i}+\hat{\phi}_{j}-\phi_{12}} / \eta_{d_{i}}$. Finally, note that from (5) we have $\eta_{d_{i}}=\eta_{\phi_{i}}$, which yields

$$
\frac{\eta_{D_{12}}}{\eta_{d_{i}}}>\frac{\eta_{\phi_{i}+\hat{\phi}_{j}-\phi_{12}}}{\eta_{\phi_{i}}}
$$

\section{Proof of Lemma 1:}

For the bivariate normal distribution with mean $(0,0)$ and variance $\Sigma=((1, \rho),(\rho, 1))$, we can write ${ }^{31}$

$$
D_{12}=\frac{1}{2 \pi \sqrt{1-\rho^{2}}} \int_{n_{2}}^{\infty} \int_{n_{1}}^{\infty} e^{-\frac{q_{1}^{2}-2 \rho q_{1} q_{2}+q_{2}^{2}}{2\left(1-\rho^{2}\right)}} d q_{1} d q_{2} .
$$

We can now perform integration with respect to $q_{1}$ and then differentiate with respect to $\rho$. Performing first the $q_{1}$ integration leads to the following expression:

$$
D_{12}=\frac{1}{2 \pi \sqrt{1-\rho^{2}}} \int_{n_{2}}^{\infty} \frac{\sqrt{\pi\left(1-\rho^{2}\right)} e^{-\frac{q_{2}^{2}}{2}}}{\sqrt{2}}\left[\operatorname{erf}\left(\frac{\rho q_{2}-n_{1}}{\sqrt{2\left(1-\rho^{2}\right)}}\right)-\lim _{q_{1} \rightarrow \infty} \operatorname{erf}\left(\frac{\rho q_{2}-q_{1}}{\sqrt{2\left(1-\rho^{2}\right)}}\right)\right] d q_{2}
$$

where $\operatorname{erf}(\cdot)$ is an error function. Since $\operatorname{erf}(-\infty)=-1$, we can write the above expression as

$$
D_{12}=\frac{1}{2 \sqrt{2 \pi}} \int_{n_{2}}^{\infty} e^{-\frac{q_{2}^{2}}{2}}\left[\operatorname{erf}\left(\frac{\rho q_{2}-n_{1}}{\sqrt{2\left(1-\rho^{2}\right)}}\right)+1\right] d q_{2} .
$$

Taking the derivative with respect to $\rho$ yields

$$
\frac{\partial D_{12}}{\partial \rho}=\frac{1}{2 \pi\left(1-\rho^{2}\right)^{3 / 2}} \int_{n_{2}}^{\infty} e^{-\frac{n_{1}^{2}-2 \rho n_{1} q_{2}+q_{2}^{2}}{2\left(1-\rho^{2}\right)}}\left(q_{2}-\rho n_{1}\right) d q_{2} .
$$

\footnotetext{
${ }^{31}$ To simplify the exposition, we set $\gamma=1$ in the proofs of Lemmas 1 and 2 and Proposition 2.
} 
We can integrate the right-hand side of (18) directly to obtain

$$
\frac{\partial D_{12}}{\partial \rho}=\frac{1}{2 \pi \sqrt{1-\rho^{2}}} e^{-\frac{n_{1}^{2}-2 \rho n_{1} n_{2}+n_{2}^{2}}{2\left(1-\rho^{2}\right)}}>0
$$

It is evident that the right-hand side of the last expression is the derivative of $D_{12}$ with respect to $n_{1}$ and $n_{2}$. Because the integrand of the expression in (17) is well-behaved, we can also reverse the order of integration and differentiation to obtain the same result. As a consequence, we have $\partial D_{12} / \partial \rho>0$ for all $\left(n_{1}, n_{2}\right)$.

\section{Proof of Lemma 2:}

Taking the derivative of $\eta_{D_{12}}$ with respect to $\rho$, we obtain

$$
\frac{\partial \eta_{D_{12}}}{\partial \rho}=-\frac{\partial\left(\frac{\partial D_{12}}{\partial n_{i}}\right)}{\partial \rho} \frac{n_{i}}{D_{12}}+\frac{\partial D_{12}}{\partial \rho} \frac{\partial D_{12}}{\partial n_{i}} \frac{n_{i}}{D_{12}^{2}}=-\frac{n_{i}}{D_{12}}\left(\frac{\partial^{2} D_{12}}{\partial n_{i} \partial \rho}-\frac{\partial D_{12}}{\partial \rho} \frac{\partial D_{12}}{\partial n_{i}} \frac{1}{D_{12}}\right) \text {. }
$$

Rearranging (19) and using $\partial D_{12} / \partial \rho>0$ yields that $\partial \eta_{D_{12}} / \partial \rho<0$ if and only if

$$
\frac{\frac{\partial D_{12}}{\partial n_{i}}}{D_{12}}<\frac{\frac{\partial^{2} D_{12}}{\partial n_{i} \partial \rho}}{\frac{\partial D_{12}}{\partial \rho}}
$$

We can write $D_{12}$ as $D_{12}=\int_{n_{2}}^{\infty} \int_{n_{1}}^{\infty} h\left(q_{1}, q_{2}\right) d q_{2} d q_{1}$, where $h\left(q_{1}, q_{2}\right)$ is the probability density function of the reservation values $\left(q_{1}, q_{2}\right)$. As we have shown in the proof of the last lemma, for the bivariate normal distribution, $\partial D_{12} / \partial \rho$ is the derivative of $D_{12}$ with respect to $n_{1}$ and $n_{2}$. This implies

$$
\frac{\partial D_{12}}{\partial \rho}=h\left(n_{1}, n_{2}\right)
$$

We can therefore rewrite $(20)$ as

$$
-\frac{\int_{n_{j}}^{\infty} h\left(n_{i}, q_{j}\right) d q_{j}}{\int_{n_{2}}^{\infty} \int_{n_{1}}^{\infty} h\left(q_{1}, q_{2}\right) d q_{2} d q_{1}}<\frac{\frac{\partial h\left(n_{1}, n_{2}\right)}{\partial n_{i}}}{h\left(n_{1}, n_{2}\right)} .
$$

We note here that due to the fact that $\partial D_{12} / \partial \rho=h\left(n_{1}, n_{2}\right)$, condition (20) is equivalent to

$$
\frac{\frac{\partial^{2} D_{12}}{\partial n_{i} \partial n_{j}}}{D_{12}}
$$

increasing in $n_{i}$. This is a two-dimensional monotone hazard rate condition. Specifically, it is equivalent to $h\left(n_{1}, n_{2}\right) / \bar{H}\left(n_{1}, n_{2}\right)$ increasing in $n_{1}$ and $n_{2}$, where $\bar{H} \equiv D_{12}$ is the survival function and $h$ is the probability density function.

Using the fact that $h\left(n_{1}, n_{2}\right)=1 /\left(2 \pi \sqrt{1-\rho^{2}}\right) e^{-\frac{n_{1}^{2}-2 \rho n_{1} n_{2}+n_{2}^{2}}{2\left(1-\rho^{2}\right)}}$, we can rewrite the right hand-side of (21) as

$$
-\frac{n_{i}-\rho n_{j}}{1-\rho^{2}}
$$

Now we turn to the left-hand side of $(21)$. The numerator is $\int_{n_{j}}^{\infty} h\left(n_{i}, q_{j}\right) d q_{j}$. We can rearrange this 
to

$$
\begin{gathered}
\int_{n_{j}}^{\infty} h\left(n_{i}, q_{j}\right) d q_{j}=\int_{n_{j}}^{\infty} \frac{1}{2 \pi \sqrt{1-\rho^{2}}} e^{-\frac{n_{i}^{2}-2 \rho n_{i} q_{j}+q_{j}^{2}}{2\left(1-\rho^{2}\right)}} d q_{j} \\
=\left|\int_{n_{j}}^{\infty} \frac{1}{2 \pi \sqrt{1-\rho^{2}}} e^{-\frac{q_{i}^{2}-2 \rho q_{i} q_{j}+q_{j}^{2}}{2\left(1-\rho^{2}\right)}} d q_{j}\right|_{\infty}^{n_{i}} \\
=\int_{n_{j}}^{\infty} \int_{\infty}^{n_{i}} \frac{1}{2 \pi \sqrt{1-\rho^{2}}} \frac{\partial e^{-\frac{q_{i}^{2}-2 \rho q_{i} q_{j}+q_{j}^{2}}{2\left(1-\rho^{2}\right)}}}{\partial q_{i}} d q_{i} d q_{j} \\
=\int_{n_{j}}^{\infty} \int_{\infty}^{n_{i}} \frac{1}{2 \pi \sqrt{1-\rho^{2}}} \frac{\rho q_{j}-q_{i}}{1-\rho^{2}} e^{-\frac{q_{i}^{2}-2 \rho q_{i} q_{j}+q_{j}^{2}}{2\left(1-\rho^{2}\right)}} d q_{i} d q_{j} \\
=\int_{n_{j}}^{\infty} \int_{n_{i}}^{\infty} \frac{q_{i}-\rho q_{j}}{2 \pi \sqrt{1-\rho^{2}}\left(1-\rho^{2}\right)} e^{-\frac{q_{i}^{2}-2 \rho q_{i} q_{j}+q_{j}^{2}}{2\left(1-\rho^{2}\right)}} d q_{i} d q_{j} .
\end{gathered}
$$

The denominator is given by

$$
\int_{n_{j}}^{\infty} \int_{n_{i}}^{\infty} h\left(q_{1}, q_{2}\right) d q_{2} d q_{1}=\int_{n_{j}}^{\infty} \int_{n_{i}}^{\infty} \frac{1}{2 \pi \sqrt{1-\rho^{2}}} e^{-\frac{q_{i}^{2}-2 \rho q_{i} q_{j}+q_{j}^{2}}{2\left(1-\rho^{2}\right)}} d q_{2} d q_{1} .
$$

We can therefore write the left-hand side of (21) as

$$
-\frac{\int_{n_{j}}^{\infty} \int_{n_{i}}^{\infty}\left(q_{i}-\rho q_{j}\right) e^{-\frac{q_{i}^{2}-2 \rho q_{i} q_{j}+q_{j}^{2}}{2\left(1-\rho^{2}\right)}} d q_{i} d q_{j}}{\int_{n_{j}}^{\infty} \int_{n_{i}}^{\infty} e^{-\frac{q_{i}^{2}-2 \rho q_{i} q_{j}+q_{j}^{2}}{2\left(1-\rho^{2}\right)}} d q_{j} d q_{i}} \frac{1}{1-\rho^{2}} .
$$

For $n_{j}=n_{i}$ we can rewrite this as

$$
-\frac{\int_{n_{j}}^{\infty} \int_{n_{i}}^{\infty} q_{j} e^{-\frac{q_{i}^{2}-2 \rho q_{i} q_{j}+q_{j}^{2}}{2\left(1-\rho^{2}\right)}} d q_{i} d q_{j}}{\int_{n_{j}}^{\infty} \int_{n_{i}}^{\infty} e^{-\frac{q_{i}^{2}-2 \rho q_{i} q_{j}+q_{j}^{2}}{2\left(1-\rho^{2}\right)}} d q_{j} d q_{i}} \frac{1-\rho}{1-\rho^{2}}=-E\left(q_{j} \mid q_{j} \geq n_{j}, q_{i} \geq n_{i}\right) \frac{1-\rho}{1-\rho^{2}}
$$

For $n_{i}=n_{j}$, the right hand-side of (21) is given by

$$
-n_{j} \frac{1-\rho}{1-\rho^{2}}
$$

Since

$$
-E\left(q_{j} \mid q_{j} \geq n_{j}, q_{i} \geq n_{i}\right) \frac{1-\rho}{1-\rho^{2}}<-n_{j} \frac{1-\rho}{1-\rho^{2}} .
$$

the inequality in (21) is always fulfilled, implying that $\partial \eta_{D_{12}} / \partial \rho<0$.

\section{Proof of Proposition 2:}

First, note that $\eta_{d_{i}}$ is unaffected by $\rho$. Hence, the left-hand side of (7) is strictly decreasing in $\rho$. Now let us look at the case $\rho=0$. The left-hand side of (7) is given by $\eta_{D_{12}} / \eta_{d_{i}}$. The denominator is 
given by

$$
\eta_{d_{i}}=\frac{e^{-\frac{n_{i}^{2}}{2}}}{\int_{n_{i}}^{\infty} e^{-\frac{q_{i}^{2}}{2}} d q_{i}} n_{i}
$$

while the numerator is given by

$$
\eta_{D_{12}}=\frac{\int_{n_{j}}^{\infty} e^{-\frac{n_{i}^{2}+q_{j}^{2}}{2}} d q_{j}}{\int_{n_{j}}^{\infty} \int_{n_{i}}^{\infty} e^{-\frac{q_{i}^{2}+q_{j}^{2}}{2}} d q_{i} d q_{j}} n_{i}
$$

For $n_{j}=n_{i}$, the last equation can be written as

$$
\eta_{D_{12}}=\frac{e^{-\frac{n_{i}^{2}}{2}} \int_{n_{i}}^{\infty} e^{-\frac{q_{i}^{2}}{2}} d q_{i}}{\left(\int_{n_{i}}^{\infty} e^{-\frac{q_{i}^{2}}{2}} d q_{i}\right)^{2}}
$$

Dividing (23) by (22), it is easy to see that this equals 1 , which implies that $\eta_{D_{12}} / \eta_{d_{i}}=1$ at $\rho=0$.

Finally, it is readily checked that for $n_{i}=n_{j}$, we have $\eta_{\phi_{i}+\phi_{j}-\phi_{12}} / \eta_{\phi_{i}}=1$, implying that the righthand side of (7) is equal to 1 , independent of $\rho$. This result coupled with the fact that the left-hand side equals 1 at $\rho=0$ and that it is strictly decreasing in $\rho$ yields the result.

\section{Proof of Proposition 3:}

We know that $n_{i}^{d}>n_{i}^{m}$ if and only if

$$
1>\frac{\eta_{\hat{\phi}_{i} \hat{\phi}_{j}+\Delta_{\phi_{i}}}}{\eta_{\phi_{i}}}=\frac{\eta_{\hat{\phi}_{i} \hat{\phi}_{j}+\phi_{i}-\hat{\phi}_{i}}}{\eta_{\phi_{i}}}
$$

or

$$
\eta_{\phi_{i}}>\eta_{\hat{\phi}_{i} \hat{\phi}_{j}+\phi_{i}-\hat{\phi}_{i}}
$$

Writing out the respective expressions for the elasticities gives

$$
\frac{\partial \phi_{i}}{\partial n_{i}} \frac{n_{i}}{\phi_{i}}>\frac{\partial\left(\phi_{i}-\hat{\phi}_{i}+\hat{\phi}_{i} \hat{\phi}_{j}\right)}{\partial n_{i}} \frac{n_{i}}{\phi_{i}-\hat{\phi}_{i}+\hat{\phi}_{i} \hat{\phi}_{j}} .
$$

Since $\partial \hat{\phi}_{j} / \partial n_{i}=0$, we have $\partial\left(\hat{\phi}_{i} \hat{\phi}_{j}\right) / \partial n_{i}=\hat{\phi}_{j}\left(\partial \hat{\phi}_{i}\right) / \partial n_{i}$. Inserting this into (24) and rearranging yields

$$
\frac{\partial \phi_{i}}{\partial n_{i}}\left(\frac{n_{i}}{\phi_{i}}-\frac{n_{i}}{\phi_{i}-\hat{\phi}_{i}+\hat{\phi}_{i} \hat{\phi}_{j}}\right)>-\left(1-\hat{\phi}_{j}\right) \frac{\partial \hat{\phi}_{i}}{\partial n_{i}} \frac{n_{i}}{\phi_{i}-\hat{\phi}_{i}+\hat{\phi}_{i} \hat{\phi}_{j}}
$$

Simplifying and dividing $(25)$ by $\hat{\phi}_{i}\left(\hat{\phi}_{j}-1\right)<0$ yields

$$
\frac{\partial \phi_{i}}{\partial n_{i}} \frac{n_{i}}{\phi_{i}}<\frac{\partial \hat{\phi}_{i}}{\partial n_{i}} \frac{n_{i}}{\hat{\phi}_{i}}
$$

or

$$
\eta_{\phi_{i}}<\eta_{\hat{\phi}_{i}}
$$




\section{Proof of Proposition 4:}

Consider first the case of competing outlets. From (3), we know that outlet $i$ 's profit maximization problem is

$$
\max _{n_{i}} \Pi_{i}^{d}=\left[D_{i}\left(n_{i}, n_{j}\right) \phi_{i}\left(n_{i}\right)+D_{12}\left(n_{i}, n_{j}\right)\left(\phi_{12}\left(n_{i}, n_{j}\right)-\hat{\phi}_{j}\left(n_{j}\right)\right)\right] .
$$

The equilibrium advertising levels are therefore characterized by the following system of first-order conditions (arguments omitted):

$$
\frac{\partial D_{i}}{\partial n_{i}} \phi_{i}+D_{i} \frac{\partial \phi_{i}}{\partial n_{i}}+\frac{\partial D_{12}}{\partial n_{i}}\left(\phi_{12}-\hat{\phi}_{j}\right)+D_{12} \frac{\partial \phi_{12}}{\partial n_{i}}=0, \quad i, j=1,2 ; j=3-i
$$

Consider now the case of joint ownership. The joint monopolist's problem is

$$
\max _{n_{i}, n_{j}} \Pi^{j o}=D_{1} \phi_{1}+D_{2} \phi_{2}+D_{12} \phi_{12}, \quad i, j=1,2 ; j=3-i .
$$

Taking the first-order condition of (28) with respect to $n_{i}$ we obtain

$$
\frac{\partial D_{i}}{\partial n_{i}} \phi_{i}+D_{i} \frac{\partial \phi_{i}}{\partial n_{i}}+\frac{\partial D_{j}}{\partial n_{i}} \phi_{j}+\frac{\partial D_{12}}{\partial n_{i}} \phi_{12}+D_{12} \frac{\partial \phi_{12}}{\partial n_{i}}=0, \quad i, j=1,2 ; j=3-i .
$$

After using $\partial D_{j} / \partial n_{i}=-\partial D_{12} / \partial n_{i}$, we can rewrite $(29)$ to

$$
\frac{\partial D_{i}}{\partial n_{i}} \phi_{i}+D_{i} \frac{\partial \phi_{i}}{\partial n_{i}}+\frac{\partial D_{12}}{\partial n_{i}}\left(\phi_{12}-\phi_{j}\right)+D_{12} \frac{\partial \phi_{12}}{\partial n_{i}}=0, \quad i, j=1,2 ; j=3-i .
$$

Comparing (30) with (27), it is evident that at $n_{i}=n_{i}^{d}$, (30) is positive if and only if $\hat{\phi}_{j}<\phi_{j}$. This implies that $n_{i}^{j o}>n_{i}^{d}$ if and only if $\phi_{j}>\hat{\phi}_{j}$ and $n_{i}^{j o}=n_{i}^{d}$ if and only if $\phi_{j}=\hat{\phi}_{j}$.

\section{Proof of Proposition 5:}

We first look at the last three terms in $W$, i.e., $D_{1} \phi_{1}+D_{2} \phi_{2}+D_{12} \phi_{12}$. Taking the derivative of these terms gives

$$
\frac{\partial D_{j}}{\partial n_{j}} \phi_{i}+D_{j} \frac{\partial \phi_{j}}{\partial n_{j}}+\frac{\partial D_{i}}{\partial n_{j}} \phi_{i}+\frac{\partial D_{12}}{\partial n_{j}} \phi_{12}+D_{12} \frac{\partial \phi_{12}}{\partial n_{j}}
$$

We can now substitute $\partial D_{12} / \partial n_{j}=-\partial D_{i} / \partial n_{j}$ into (31) to obtain

$$
\frac{\partial D_{j}}{\partial n_{j}} \phi_{j}+D_{j} \frac{\partial \phi_{j}}{\partial n_{j}}+\frac{\partial D_{12}}{\partial n_{j}}\left(\phi_{12}-\phi_{i}\right)+D_{12} \frac{\partial \phi_{12}}{\partial n_{j}} .
$$

It is evident that this expression is equivalent to (30). Therefore, at $n_{j}=n_{j}^{j o}$, this expression equals zero. Since $n_{j}^{d}=n_{j}^{j o}$ for $\phi_{j}=\hat{\phi}_{j}$, the last three terms of $W$ are maximized at $n_{j}=n_{j}^{d}$.

However, the first terms in $W$ are the utilities of the viewers which are strictly decreasing in $n_{j}$. As a consequence, the first-order condition of $W$ with respect to $n_{j}$ evaluated at $n_{j}=n_{j}^{d}$ is strictly negative, which implies that there is too much advertising in duopoly at $\phi_{j}=\hat{\phi}_{j}$. By continuity, this result also holds for $\hat{\phi}_{j}$ close to $\phi_{j}$. 


\subsection{Further Material}

\section{Two-stage game}

Consider the following assumptions:

A1 Outlets are symmetric.

A2 For any $\alpha \in[0,1]$, the following inequality holds

$$
t_{i}^{\star}(1-\alpha)>\alpha\left\{d_{i}\left(\alpha n_{i}^{d}\right) \phi\left(n_{i}^{d}\right)-d_{i}\left((1-\alpha) n_{i}^{\star}\right) \phi\left(n_{i}^{\star}\right)\right\}
$$

where $d_{i}(\cdot):=D_{i}(\cdot)+D_{12}(\cdot), n_{i}^{d}=\arg \max _{n_{i}} d_{i}\left(\alpha n_{i}\right) \phi\left(n_{i}\right), n_{i}^{\star}$ is implicitly defined by $(6)$ and $t_{i}^{\star}$ is given by $D_{i}\left(n_{i}^{\star}, n_{j}^{\star}\right) \phi_{i}\left(n_{i}^{\star}\right)+D_{12}\left(n_{i}^{\star}, n_{j}^{\star}\right)\left(\phi_{12}\left(n_{i}^{\star}, n_{j}^{\star}\right)-\hat{\phi}_{j}\left(n_{j}^{\star}\right)\right)$.

We provide a discussion of these assumptions after the proof of the following proposition. There we explain that $A 1$ can be weakened while $A 2$ is a relatively natural assumption in our framework.

Proposition Suppose that $A 1$ and $A 2$ hold. Then, there is an equilibrium in the two-stage game game with posted contracts, that is outcome-equivalent to the equilibrium of the game defined in Section 2 .

\section{Proof:}

Suppose that in the two-stage game with posted contracts each outlet offers a contract with $n_{i}=n_{i}^{\star}$, where $n_{i}^{\star}$ is implicitly defined by $(6)$, and a transfer

$$
t_{i}^{\star}=D_{i}\left(n_{i}^{\star}, n_{j}^{\star}\right) \phi_{i}\left(n_{i}^{\star}\right)+D_{12}\left(n_{i}^{\star}, n_{j}^{\star}\right)\left(\phi_{12}\left(n_{i}^{\star}, n_{j}^{\star}\right)-\hat{\phi}_{j}\left(n_{j}^{\star}\right)\right) .
$$

By the same argument as we used for the original model, these contracts will be accepted by all advertisers. As this is anticipated by viewers, viewerships are $D_{i}\left(n_{i}^{\star}, n_{j}^{\star}\right)$ and $D_{12}\left(n_{i}^{\star}, n_{j}^{\star}\right)$. Since advertising levels are the same as in the equilibrium of the original model, viewerships are also the same. Therefore, this candidate equilibrium is outcome-equivalent to the equilibrium of the original model.

Let us now consider if there exists a profitable deviation from this candidate equilibrium. We first show that there can be no profitable deviation contract of outlet $i$ that still induces full advertiser participation on outlet $j$ but a smaller participation on outlet $i$. Let $x_{i}$ denote the fraction of advertisers who accept the offer of outlet $i$.

Consider a candidate contract $\left(n_{i}, t_{i}\right)$. Suppose that outlet $i$ 's equilibrium profit from this contract is $t_{i} x_{i}$. Now consider the following alternative contract: $\left(x_{i} n_{i}, x_{i} t_{i}\right)$. Note that total advertising on outlet $i$ is still equal to $x_{i} n_{i}$. So outlet $i$ is at least as attractive as with the candidate equilibrium contract. Note moreover that because $\phi_{i}$ and $\phi_{12}$ are strictly concave in $n_{i}$, the incremental value of accepting offer $\left(x_{i} n_{i}, x_{i} t_{i}\right)$ must exceed $x_{i} t_{i}$ for all levels of advertiser participation. So all advertisers would accept $\left(x_{i} n_{i}, x_{i} t_{i}\right)$ regardless. It follows that outlet $i$ can marginally increase $x_{i} t_{i}$ while still getting full participation. Therefore, profits would strictly increase. It follows that no offer inducing a level of participation $x_{i}<1$ can be part of a best reply.

Now suppose outlet $i$ deviates from the candidate equilibrium in such a way that it induces a fraction $\alpha$ of the advertisers to single-home on its outlet while the remaining fraction $1-\alpha$ single-homes on outlet $j$. Using the definition $d_{i}(\cdot):=D_{i}(\cdot)+D_{12}(\cdot)$, the largest possible transfer that outlet $i$ can ask is then bounded above by

$$
t_{i}^{d}=d_{i}\left(\alpha n_{i}^{d}\right) \phi_{i}\left(n_{i}^{d}\right)-u_{s h j}
$$


where $n_{i}^{d}$ denotes the optimal deviation advertising level and $u_{s h j}$ denotes the payoff of an advertiser who chooses to reject the contract of outlet $i$ and instead single-homes on outlet $j$. To determine $u_{s h j}$ we determine the advertiser's payoff when accepting only outlet $j$ 's contract, which is the outlet's equilibrium contract after outlet $i$ has deviated to induce a fraction $\alpha$ of advertisers to single-home on outlet $i$. We obtain

$$
\begin{gathered}
u_{s h j}=d_{j}\left((1-\alpha) n_{j}^{\star}, \alpha n_{i}^{d}\right) \phi_{j}\left(n_{j}^{\star}\right)-t_{j}^{\star}= \\
d_{j}\left((1-\alpha) n_{j}^{\star}, \alpha n_{i}^{d}\right) \phi_{j}\left(n_{j}^{\star}\right)-D_{j}\left(n_{j}^{\star}, n_{i}^{\star}\right) \phi_{j}\left(n_{j}^{\star}\right)-D_{12}\left(n_{i}^{\star}, n_{j}^{\star}\right)\left(\phi_{12}\left(n_{i}^{\star}, n_{j}^{\star}\right)-\hat{\phi}_{i}\left(n_{i}^{\star}\right)\right) .
\end{gathered}
$$

Outlet $i$ 's profit is then $\alpha t_{i}^{d}$. Hence, deviating is not profitable if

$$
\begin{aligned}
& \alpha\left\{d_{i}\left(\alpha n_{i}^{d}\right) \phi_{i}\left(n_{i}^{d}\right)-\right.\left.d_{j}\left((1-\alpha) n_{j}^{\star}\right) \phi_{j}\left(n_{j}^{\star}\right)+D_{j}\left(n_{j}^{\star}, n_{i}^{\star}\right) \phi_{j}\left(n_{j}^{\star}\right)+D_{12}\left(n_{i}^{\star}, n_{j}^{\star}\right)\left(\phi_{12}\left(n_{i}^{\star}, n_{j}^{\star}\right)-\hat{\phi}_{i}\left(n_{i}^{\star}\right)\right)\right\} \\
&<D_{i}\left(n_{i}^{\star}, n_{j}^{\star}\right) \phi_{i}\left(n_{i}^{\star}\right)+D_{12}\left(n_{i}^{\star}, n_{j}^{\star}\right)\left(\phi_{12}\left(n_{i}^{\star}, n_{j}^{\star}\right)-\hat{\phi}_{j}\left(n_{j}^{\star}\right)\right) .
\end{aligned}
$$

Now suppose that the two outlets are symmetric. Then the above condition reduces to

$\alpha\left\{d_{i}\left(\alpha n^{d}\right) \phi\left(n^{d}\right)-d_{i}\left((1-\alpha) n^{\star}\right) \phi\left(n^{\star}\right)\right\}-(1-\alpha)\left(D_{i}\left(n^{\star}, n^{\star}\right) \phi\left(n^{\star}\right)+D_{12}\left(n^{\star}, n^{\star}\right)\left(\phi_{12}\left(n^{\star}, n^{\star}\right)-\hat{\phi}\left(n^{\star}\right)\right)\right)<0$,

where $n_{i}^{\star}=n_{j}^{\star}=n^{\star}, n_{i}^{d}=n^{d}, \hat{\phi}_{i}(\cdot)=\hat{\phi}_{j}(\cdot)=\hat{\phi}(\cdot)$, and $\phi_{i}(\cdot)=\phi_{j}(\cdot)=\phi(\cdot)$. This can be rewritten as

$$
t_{i}^{\star}(1-\alpha)>\alpha\left\{d_{i}\left(\alpha n_{i}^{d}\right) \phi\left(n_{i}^{d}\right)-d_{i}\left((1-\alpha) n_{i}^{\star}\right) \phi\left(n_{i}^{\star}\right)\right\}
$$

which is fulfilled by $A 2$. As a consequence, a deviation is not profitable.

We now shortly explain why the assumptions $A 1$ and $A 2$ are not very restrictive in our framework. First, consider $A 1$. Since the game is continuous, $A 1$ can be relaxed to some extent without affecting the result, implying that the proposition still holds if outlets are not too asymmetric. Now consider $A 2$. It is evident from (32), that the assumption is fulfilled for $\alpha$ low enough. In this case the right-hand side is close to 0 , while the left-hand side is strictly positive. Now consider the opposite case, i.e., $\alpha \rightarrow 1$. In that case the left-hand side goes to zero, while the right-hand side goes to $d_{i}\left(n_{i}^{d}\right) \phi\left(n_{i}^{d}\right)-d_{i}(0) \phi\left(n_{i}^{\star}\right)$. Evidently, $d_{i}(0)>d_{i}\left(n_{i}^{d}\right)$. Hence, the right-hand side is negative if $\phi\left(n_{i}^{d}\right)$ is not much larger than $\phi\left(n_{i}^{\star}\right)$. In general, $n_{i}^{\star}$ can be larger or smaller than $n_{i}^{d}$, implying that the difference can be either positive or negative. However, even in case $n_{i}^{d}>n_{i}^{\star}$, if the slope of the advertising functions $\phi_{i}$ and $\phi_{12}$ is relatively small, we find that the difference between $n_{i}^{\star}$ and $n_{i}^{d}$ is small and so the right-hand side is negative. Finally, consider intermediate values of $\alpha$. Again, if the difference between $n_{i}^{\star}$ and $n_{i}^{d}$ is relatively small, the term in the bracket on the right-hand side of (32) is close to zero. Since the left-hand side is strictly positive, $A 2$ is then fulfilled as well.

\section{Entry in case of two incumbent outlets}

Consider the case of two incumbents and entry of a third outlet. After entry, the profit of outlet $i$ is

$$
\begin{gathered}
\left.\Pi_{(} n_{1}, n_{2}, n_{3}\right)=D_{i}\left(n_{1}, n_{2}, n_{3}\right) \phi_{i}\left(n_{i}\right)+D_{i j}\left(n_{1}, n_{2}, n_{3}\right)\left(\phi_{i j}\left(n_{i}, n_{j}\right)-\hat{\phi}_{j}\left(n_{j}\right)\right) \\
+D_{i k}\left(n_{1}, n_{2}, n_{3}\right)\left(\phi_{i k}\left(n_{i}, n_{k}\right)-\hat{\phi}_{k}\left(n_{k}\right)\right)+D_{123}\left(n_{1}, n_{2}, n_{3}\right)\left(\phi_{i j k}\left(n_{i}, n_{j}, n_{k}\right)-\phi_{j k}\left(n_{j}, n_{k}\right)\right)
\end{gathered}
$$


As in the case of entry of a second outlet, we can rewrite this profit function as the profit without entry plus a negative correction term. This leads to (dropping arguments)

$$
\begin{gathered}
\Pi=\left(D_{i}+D_{i k}\right) \phi_{i}+\left(D_{i j}+D_{i j k}\right)\left(\phi_{i j}-\hat{\phi}_{j}\right) \\
-D_{i k}\left(\phi_{i}+\hat{\phi}_{k}-\phi_{i k}\right)-D_{i j k}\left(\phi_{i j}-\hat{\phi}_{j}-\left(\phi_{i j k}-\phi_{j k}\right)\right) .
\end{gathered}
$$

The first two terms are the profit in duopoly. Note that without entry $D_{i k}$ did not exist since there was no outlet $k$ and so outlet $i$ could get $\phi_{i}$ for these viewers due to the fact that they were single-homing on outlet $i$. Similarly, $D_{i j k}$ did not exist and these viewers were multi-homing in outlets $i$ and $j$. The last two terms are the negative correction terms.

Taking the derivative with respect to $n_{i}$ yields

$\frac{\partial \Pi}{\partial n_{i}}=\frac{\partial \Pi^{d}}{\partial n_{i}}+D_{i k}\left(\phi_{i}+\hat{\phi}_{k}-\phi_{i k}\right)\left[\eta_{D_{i k}}-\eta_{\phi_{i}+\hat{\phi}_{k}-\phi_{i k}}\right]+D_{i j k}\left(\phi_{i j}-\hat{\phi}_{j}-\left(\phi_{i j k}-\phi_{j k}\right)\right)\left[\eta_{D_{i j k}}-\eta_{\phi_{i j}-\hat{\phi}_{j}-\left(\phi_{i j k}-\phi_{j k}\right)}\right]=0$.

So we obtain that for $\eta_{D_{i k}}>\eta_{\phi_{i}+\phi_{k}-\phi_{i k}}$ and $\eta_{D_{i j k}}>\eta_{\phi_{i j}-\phi_{j}-\left(\phi_{i j k}-\phi_{j k}\right)}$, the business-sharing effect dominates the duplication effect. The formula now consists of two terms since entry of a third outlet leads to changes in two viewer groups, namely, the exclusive ones and the overlapping ones before entry. Each term is multiplied by the absolute profits of the respective viewer group. This analysis can be extended to any number of incumbent outlets.

\section{Equilibrium with Heterogeneous Advertisers}

We first determine the solution to the more complicated duopoly problem. (Solving the monopoly problem proceeds along very similar lines and we will describe it very briefly towards the end.) The problem of a duopolist $i$ is to maximize its profits $\int_{\underline{\omega}}^{\bar{\omega}} t_{i}\left(m_{i}(\omega)\right) d F(\omega)$ with respect to the transfer schedule, given its rival's choice $t_{j}\left(m_{j}\right)$. From the main text, this problem can be rewritten as in (14). Denote by $m_{j}^{\star}(m, \omega)$ the advertising intensity that type $\omega$ optimally buys from outlet $j$ when buying intensity $m$ from outlet $i$. Then, the net contracting surplus for type $\omega$ is

$$
\begin{aligned}
v_{i}^{d}(m, \omega, n)= & \max _{y}\left[\omega u(m, y, n)-t_{j}(y)\right]-\left(\max _{y^{\prime}}\left[\omega u\left(0, y^{\prime}, n\right)-t_{j}\left(y^{\prime}\right)\right]\right) \\
& =\omega u\left(m, m_{j}^{\star}(m, \omega), n\right)-t_{j}\left(m_{j}^{\star}(m, \omega)\right)-\omega u\left(0, m_{j}^{\star}(0, \omega), n\right)+t_{j}\left(m_{j}^{\star}(0, \omega)\right)
\end{aligned}
$$

Incentive compatibility requires $m_{i}(\omega)=\arg \max _{m} v_{i}^{d}(m, \omega, n)-t_{i}(m)$, which implies

$$
v_{i}^{d}\left(m_{i}(\omega), \omega, n\right)-t_{i}\left(m_{i}(\omega)\right)=\max _{y, y^{\prime}, m}\left\{\omega u(m, y, n)-t_{j}(y)-\left(\omega u\left(0, y^{\prime}, n\right)-t_{j}\left(y^{\prime}\right)\right)-t_{i}(m)\right\}
$$

By the envelope theorem the derivative of the above with respect to $\omega$ is

$$
u\left(m, m_{j}^{\star}\left(n_{i}(\omega), \omega\right), n\right)-u\left(0, m_{j}^{\star}(0, \omega), n\right)
$$

Since this pins down the growth rate of the advertiser's payoff, we find that $\max _{\omega_{0}^{i}, m_{i}(\cdot)} \int_{\omega_{0}^{i}}^{\bar{\omega}} t_{i}\left(m_{i}(\omega)\right)$ 
subject to the first two constraints of (12) equals

$$
\begin{aligned}
\max _{\omega_{0}^{i}, m_{i}(\cdot)} \int_{\omega_{0}}^{\bar{\omega}}\left\{\omega u\left(m_{i}(\omega), m_{j}^{\star}\left(m_{i}(\omega), \omega\right), n\right)-\omega u\left(0, m_{j}^{\star}(0, \omega), n\right)-t_{j}\left(m_{j}^{\star}\left(m_{i}(\omega), \omega\right)\right)+t_{j}\left(m_{j}^{\star}(0, \omega)\right)\right. \\
\left.-\int_{\omega_{0}^{i}}^{\omega}\left[\omega u\left(m, m_{j}^{\star}\left(m_{i}(z), z\right), n\right)-\omega u\left(0, m_{j}^{\star}(0, z), n\right)\right] d z\right\} d F(\omega) \\
=\max _{\omega_{0}^{i}, m_{i}(\cdot)} \int_{\omega_{0}^{i}}^{\bar{\omega}}\{v_{i}^{d}\left(m_{i}, \omega, n\right)-\underbrace{\left.\int_{\omega_{0}^{i}}^{\omega}\left[\omega u\left(m, m_{j}^{\star}\left(m_{i}(z), z\right), n\right)-\omega u\left(0, m_{j}^{\star}(0, z), n\right)\right] d z\right\}}_{\text {information rent }} d F(\omega),
\end{aligned}
$$

Integrating the double integral by parts gives

$$
\begin{gathered}
\max _{m_{i}(\cdot), \omega_{0}^{i}} \int_{\omega_{0}^{i}}^{\bar{\omega}} \omega u\left(m_{i}(\omega), m_{j}^{\star}\left(m_{i}(\omega), \omega\right), n\right)-\omega u\left(0, m_{j}^{\star}(0, \omega), n\right)-t_{j}\left(m_{j}^{\star}\left(m_{i}(\omega), \omega\right)\right)+t_{j}\left(m_{j}^{\star}(0, \omega)\right)+ \\
-\frac{1-F(\omega)}{f(\omega)}\left(u\left(m, m_{j}^{\star}\left(m_{i}(\omega), \omega\right), n\right)-u\left(0, m_{j}^{\star}(0, \omega), n\right)\right) d F(\omega)
\end{gathered}
$$

The duopolist's best-reply allocation of advertising intensities $m_{i}^{d}(\omega)$ then solves

$$
\begin{aligned}
\max _{m_{i}(\cdot), \omega_{0}^{i}} & \int_{\omega_{0}^{i}}^{\bar{\omega}}\left(\omega-\frac{1-F(\omega)}{f(\omega)}\right)\left(u\left(m_{i}(\omega), m_{j}^{\star}\left(m_{i}(\omega), \omega\right), n\right)-u\left(0, m_{j}^{\star}(0, \omega), n\right)\right) \\
& -\left(t_{j}\left(m_{j}^{\star}\left(m_{i}(\omega), \omega\right)\right)-t_{j}\left(m_{j}^{\star}(0, \omega)\right)\right) d F(\omega), \\
\text { subject to } & \int_{\omega_{0}^{i}}^{\bar{\omega}} m_{i}\left(\omega^{\prime}\right) d F\left(\omega^{\prime}\right) \leq n_{i} .
\end{aligned}
$$

From now on we will denote the integrand function by $\Lambda^{d}\left(m_{i}(\omega), \omega, n\right)$. Recall that solving a canonical screening problem usually involves maximizing the integral over all served types, where the integrand is the utility of type $\omega$ minus his information rent, expressed as a function of the allocation. The utility here is the incremental value $u\left(m_{i}(\omega), m_{j}^{\star}\left(m_{i}(\omega), \omega\right), n\right)-u\left(0, m_{j}^{\star}(0, \omega), n\right)$, minus the difference in transfers.

The maximization problem in the first stage with respect to $n_{i}$ can be written as

$$
\max _{n_{i}}\left(\max _{m_{i}(\cdot), \omega_{0}} \int_{\omega_{0}^{i}}^{\bar{\omega}} \Lambda^{d}\left(m_{i}(\omega), \omega, n\right) d F(\omega) \quad \text { s.t. } \quad n_{i}=\int_{\omega_{0}^{i}}^{\bar{\omega}} m_{i}(\omega) d F(\omega)\right) .
$$

Let us first determine $u\left(m_{i}(\omega), m_{j}^{\star}\left(m_{i}(\omega), \omega\right), n\right)-u\left(0, m_{j}^{\star}(0, \omega), n\right)$. Abbreviating $m_{j}^{\star}\left(m_{i}(\omega), \omega\right)$ by $m_{j}^{\star}$ and $m_{j}^{\star}(0, \omega)$ by $\left(m_{j}^{\prime}\right)^{\star}$ we can write

$$
\begin{gathered}
\left.u\left(m_{i}(\omega), m_{j}^{\star}, n\right)-u\left(0,\left(m_{j}^{\prime}\right)^{\star}\right), n\right) \\
=D_{i}\left(n_{1}, n_{2}\right) \phi_{i}\left(m_{i}(\omega)\right)+D_{j}\left(n_{1}, n_{2}\right) \phi_{j}\left(m_{j}^{\star}\right)+D_{12}\left(n_{1}, n_{2}\right) \phi_{12}\left(m_{i}(\omega), m_{j}^{\star}\right) \\
-D_{j}\left(n_{1}, n_{2}\right) \phi_{j}\left(\left(m_{j}^{\prime}\right)^{\star}\right)-D_{12}\left(n_{1}, n_{2}\right) \phi_{j}\left(\left(m_{j}^{\prime}\right)^{\star}\right) \\
=d_{i}\left(n_{i}\right) \phi_{i}\left(m_{i}(\omega)\right)+D_{12}\left(n_{1}, n_{2}\right)\left(\phi_{12}\left(m_{i}(\omega), m_{j}^{\star}\right)-\phi_{i}\left(m_{i}(\omega)\right)-\hat{\phi}_{j}\left(\left(m_{j}^{\prime}\right)^{\star}\right)\right)+D_{j}\left(n_{1}, n_{2}\right)\left(\phi_{j}\left(m_{j}^{\star}\right)-\hat{\phi}_{j}\left(\left(m_{j}^{\prime}\right)^{\star}\right)\right), \\
\text { where } \phi_{12}\left(m_{i}(\omega), m_{j}^{\star}\right)=\hat{\phi}_{i}\left(m_{i}(\omega)\right)+\hat{\phi}_{j}\left(m_{j}^{\star}\right)-\hat{\phi}_{i}\left(m_{i}(\omega)\right) \hat{\phi}_{j}\left(\left(m_{j}^{\prime}\right)^{\star}\right) .
\end{gathered}
$$

Adapting results from Martimort and Stole (2009), we know that at the optimal solution $m_{i}(\omega)=0$ for all $\omega<\omega_{0}$ and that $m_{i}(\omega)=\arg \max _{m} \Lambda^{d}\left(m_{i}(\omega), \omega, n\right)$. By our assumptions about the demand and 
advertising function, the optimal solution involves a schedule $m_{i}(\omega)$ that is non-decreasing.

From (34), we can write the maximization problem with respect to the optimal allocation of advertising intensities, given $n_{i}$, as

$$
\max _{m_{i}(\cdot), \lambda} \int_{\omega_{0}^{i}}^{\bar{\omega}} \Lambda^{d}\left(m_{i}(\omega), \omega, n\right) d F(\omega)+\lambda\left(n_{i}-\int_{\omega_{0}^{i}}^{\bar{\omega}} m_{i}(\omega) d F(\omega)\right) .
$$

Pointwise maximization with respect to $m_{i}(\cdot)$ yields

$$
\begin{gathered}
\left(\omega-\frac{1-F(\omega)}{f(\omega)}\right)\left[d_{i}\left(n_{i}\right) \frac{\partial \phi_{i}}{\partial m_{i}}+D_{12}\left(n_{1}, n_{2}\right)\left(\frac{\partial\left(\phi_{12}\left(\left(m_{i}, m_{j}^{\star}\right)\right)-\phi_{i}\left(m_{i}\right)\right)}{\partial m_{i}}\right)\right. \\
\left.+\left[D_{j}\left(n_{1}, n_{2}\right)-D_{12}\left(n_{1}, n_{2}\right)\right] \frac{\partial \phi_{j}}{\partial m_{j}^{\star}} \frac{\partial m_{j}^{\star}}{\partial m_{i}}\right]-\frac{\partial t_{j}}{\partial m_{j}^{\star}} \frac{\partial m_{j}^{\star}}{\partial m_{i}}=\lambda .
\end{gathered}
$$

Denoting the left-hand side of (35) by $\psi$, and integrating both sides from $\omega_{0}^{i}$ to $\bar{\omega}$, we obtain

$$
\frac{\int_{\omega_{0}^{i}}^{\bar{\omega}} \psi d F(\omega)}{1-F\left(\omega_{0}^{i}\right)}=\lambda
$$

The maximization problem of the first stage with respect to $n_{i}$ is

$$
\max _{m_{i}(\cdot), \lambda} \int_{\omega_{0}^{i}}^{\bar{\omega}} \Lambda_{i}^{d}\left(\omega, m_{i}(\omega)^{\star}, n_{i}\right) d F(\omega)+\lambda\left(n_{i}-\int_{\omega_{0}^{i}}^{\bar{\omega}} m_{i}(\omega)^{\star} d F(\omega)\right) .
$$

Differentiating with respect to $n_{i}$ and using the Envelope Theorem yields

$$
\begin{gathered}
\int_{\omega_{0}^{i}}^{\bar{\omega}}\left(\omega-\frac{1-F(\omega)}{f(\omega)}\right)\left[\frac{\partial d_{i}}{\partial n_{i}} \phi_{i}+\frac{\partial D_{12}}{\partial n_{i}}\left(\phi_{12}\left(\left(m_{i}, m_{j}^{\star}\right)\right)-\phi_{i}\left(m_{i}\right)-\hat{\phi}_{j}\left(\left(m_{j}^{\prime}\right)^{\star}\right)\right)\right. \\
\left.\left.+D_{12}\left[\frac{\partial \phi_{12}}{\partial m_{j}^{\star}} \frac{\partial m_{j}^{\star}}{\partial n_{i}}-\frac{\partial \hat{\phi}_{j}}{\partial\left(m_{j}^{\prime}\right)^{\star}} \frac{\partial\left(m_{j}^{\prime}\right)}{\partial n_{i}}\right]\right]+D_{j}\left[\frac{\partial \phi_{j}}{\partial m_{j}^{\star}} \frac{\partial m_{j}^{\star}}{\partial n_{i}}-\frac{\partial \hat{\phi}_{j}}{\partial\left(m_{j}^{\prime}\right)^{\star}} \frac{\partial\left(m_{j}^{\prime}\right)}{\partial n_{i}}\right]\right] d F(\omega) \\
-\frac{\partial t_{j}}{\partial m_{j}^{\star}} \frac{\partial m_{j}^{\star}}{\partial n_{i}}+\frac{\partial t_{j}}{\partial\left(m_{j}^{\prime}\right)^{\star}} \frac{\partial\left(m_{j}^{\prime}\right)^{\star}}{\partial n_{i}}=-\lambda .
\end{gathered}
$$

Combining (35) and (36) to get rid of $\lambda$ yields expression (15) of the main text, where $\kappa$ is defined as

$$
\begin{gathered}
\kappa \equiv \int_{\omega_{0}}^{\bar{\omega}}\left(\omega-\frac{1-F(\omega)}{f(\omega)}\right)\left\{\frac{1}{1-F(\omega)}\left(D_{j}-D_{12}\right) \frac{\partial \phi_{j}}{\partial m_{j}^{\star}} \frac{\partial m_{j}^{\star}}{\partial m_{i}}+D_{12}\left[\frac{\partial \phi_{12}}{\partial m_{j}^{\star}} \frac{\partial m_{j}^{\star}}{\partial n_{i}}-\frac{\partial \hat{\phi}_{j}}{\partial\left(m_{j}^{\prime}\right)^{\star}} \frac{\partial\left(m_{j}^{\prime}\right)^{\star}}{\partial n_{i}}\right]\right. \\
\left.+D_{j}\left[\frac{\partial \phi_{j}}{\partial m_{j}^{\star}} \frac{\partial m_{j}^{\star}}{\partial n_{i}}-\frac{\partial \phi_{j}}{\partial\left(m_{j}^{\prime}\right)^{\star}} \frac{\partial\left(m_{j}^{\prime}\right)^{\star}}{\partial n_{i}}\right]+\frac{\partial D_{j}}{\partial n_{i}}\left(\phi_{j}\left(m_{j}^{\star}\right)-\phi_{j}\left(\left(m_{j}^{\prime}\right)^{\star}\right)\right)-\frac{\partial t_{j}}{\partial m_{j}^{\star}} \frac{\partial m_{j}^{\star}}{\partial n_{i}}\right\} d F(\omega) \\
-\frac{\partial t_{j}}{\partial m_{j}^{\star}} \frac{\partial m_{j}^{\star}}{\partial n_{i}}+\frac{\partial t_{j}}{\partial\left(m_{j}^{\prime}\right)^{\star}} \frac{\partial\left(m_{j}^{\prime}\right)^{\star}}{\partial n_{i}} .
\end{gathered}
$$

It is evident that if outlet $j$ offers a single transfer-intensity pair, then $m_{j}^{\star}$ equals $\left(m_{j}^{\prime}\right)^{\star}$ and both are invariant to changes in $m_{i}(\cdot)$ and $n_{i}$. This implies that $\kappa=0$. 
Proceeding in the same way for the monopoly outlet, we obtain that its profit function is given by

$$
\max _{n_{i}}\left(\max _{m_{i}(\cdot), \omega_{0}^{m}} \int_{\omega_{0}^{m}}^{\bar{\omega}}\left(\omega-\frac{1-F(\omega)}{f(\omega)}\right) d_{i}\left(n_{i}\right) \phi_{i}\left(m_{i}(\omega)\right) d F(\omega) \quad \text { s.t. } \quad n_{i}=\int_{\omega_{0}^{m}}^{\bar{\omega}} m_{i}(\omega) d F(\omega)\right) .
$$

The solution is then characterized by (13).

\subsection{Empirical Analysis}

We empirically investigate the link between entry and correlation on advertising levels. As our data are limited, we regard this exercise as providing suggestive evidence, as opposed to a careful empirical analysis of the investigated issues.

The dataset is provided by Kagan-SNL a highly regarded proprietary source for information on broadcasting markets. It consists of an unbalanced panel data set of 68 basic cable channels from 1989 to 2002. The channels cover almost all cable industry advertising revenues. ${ }^{32}$ We know the date for each new network launch within our sample period (a total of 43 launches). In addition, for each network active in each year we have information on the average number of 30-second advertising slots per hour of programming (in jargon 'avails'). We also have a good coverage for other network variables, such as subscribers, programming expenses and ratings.

We first use our panel data set to study the relationship between the avails broadcasted by each channel and the number of incumbents. As our model characterizes the effects of varying competition, we consider each channel within its own competitive environment. That is, we define a relevant market segment for each of the 68 channels. The hypothesis is that channels with content tailored to the same segment compete for viewers and advertisers. For this purpose, we divide channels in three segments: (i) sports channels (henceforth Sports), (ii) channels broadcasting mainly movies and TV series (henceforth Movies\&Series), and (iii) all remaining channels, which is used as a reference group. To test whether viewer preference correlation affects the relationship between entry and advertising levels, we estimate separate parameters for the Sports and the Movies\&Series segments. Our working assumption is that the viewers' preferences within these segments are positively correlated. Our model predicts that avails would fall after entry in the Sports and Movies\&Series segments relative to the reference group. ${ }^{33,34}$

We use two different empirical approaches, a panel analysis and a model of entry episodes. We demonstrate that both lead to similar conclusions. First, we use a panel analysis, that pools all channelyear observations from 1989-2002, so it relies on within- and across-channel variation. We estimate the

\footnotetext{
${ }^{32}$ In our data, $75 \%$ of all revenues are generated by the twenty biggest networks.

${ }^{33}$ Our data does not include viewer prices. This should not be a problem because their impact was not particularly important during our sample period (see for example, Strömberg, 2004). In addition, viewer prices were highly regulated in the 1990s.

${ }^{34}$ We note that we intended to create a separate News segment as well, as this segment provides a natural counterpart to the others in that viewer preferences can be reasonably assumed to be negatively correlated. Unfortunately, the number of channels here is too small to obtain statistically meaningful results. The point estimates we obtain are nevertheless consistent with Proposition 2 (details are available from the authors).
} 
following linear regression model:

$$
\begin{aligned}
\log \left(\text { Avails }_{i t}\right)= & \beta * \text { Outlets }_{i t}+\beta_{M} * \text { Outlets }_{i t} * \text { MoviesSeries_dummy } \\
& +\beta_{S} * \text { Outlet } s_{i t} * \text { Sports_dummy }+\gamma * x_{i t}+\alpha_{i}+\delta_{t}+\epsilon_{i t},
\end{aligned}
$$

where Avails $_{i t}$ is the average number in year $t$ of 30 -second advertising slots per hour of programming by channel $i$, Outlets $s_{i t}$ is the number of channels in channel $i$ 's segment at the end of year $t$, Sports_dummy and MoviesSeries_dummy are dummy variables equal to 1 when channel $i$ belongs to the Sports and to the Movies\&Series segments, respectively, (and zero otherwise), $x_{i t}$ is a vector of channel-time controls, $\alpha_{i}$ is a channel fixed effect and $\delta_{t}$ is a time fixed effect. Given that the dependent variable is transformed in logs, while the main explanatory variable is measured in units of channels, $\beta$ has the following interpretation: when a new channel enters the control segment, the incumbents increase their 30-second advertising slots by $100 \beta \%$. The coefficients $\beta_{M}$ and $\beta_{S}$ measure the additional effect that the number of channels has on the avails in the Sports and Movies\&Series segments respectively.

\begin{tabular}{|c|c|c|c|c|c|c|}
\hline & $(1)$ & $(2)$ & $(3)$ & (4) & $(5)$ & $(6)$ \\
\hline Outlets & $\begin{array}{c}0.00268^{* *} \\
(0.00123)\end{array}$ & $\begin{array}{l}0.00104 \\
(0.00140)\end{array}$ & $\begin{array}{c}0.00974^{* * *} \\
(0.00339)\end{array}$ & $\begin{array}{c}0.0105^{* * *} \\
(0.00364)\end{array}$ & $\begin{array}{c}0.0103^{* * *} \\
(0.00362)\end{array}$ & $\begin{array}{c}0.00857^{* *} \\
(0.00407)\end{array}$ \\
\hline Real GDP & & $\begin{array}{c}0.00185 * * * \\
(0.000602)\end{array}$ & $\begin{array}{c}-0.000294 \\
(0.00134)\end{array}$ & & & \\
\hline Rev Mkt Share & & & & & $\begin{array}{l}0.178 \\
(0.255)\end{array}$ & \\
\hline Rating & & & & & & $\begin{array}{c}-0.0828 \\
(0.0769)\end{array}$ \\
\hline Constant & $\begin{array}{c}3.017^{* * *} \\
(0.0209)\end{array}$ & $\begin{array}{c}2.825^{* * *} \\
(0.0642)\end{array}$ & $\begin{array}{c}2.913^{* * *} \\
(0.112)\end{array}$ & $\begin{array}{c}2.434^{* * *} \\
(0.143)\end{array}$ & $\begin{array}{c}2.403^{* * *} \\
(0.160)\end{array}$ & $\begin{array}{c}2.969^{* * *} \\
(0.106)\end{array}$ \\
\hline Observations & 416 & 416 & 416 & 416 & 415 & 279 \\
\hline$R^{2}$ & 0.016 & 0.027 & 0.275 & 0.303 & 0.307 & 0.276 \\
\hline Channel FE & $\mathrm{NO}$ & $\mathrm{NO}$ & YES & YES & YES & YES \\
\hline Time FE & $\mathrm{NO}$ & $\mathrm{NO}$ & $\mathrm{NO}$ & YES & YES & YES \\
\hline No. of Outlets & 56 & 56 & 56 & 56 & 56 & 33 \\
\hline
\end{tabular}

Table 1: Number of Outlets and Avails - Average Effect

Table 1 reports the estimation results when we restrict the coefficient on the number of channels to be homogeneous across segments. We find evidence that entry is associated with an increase in the advertising levels on incumbent channels. The coefficient is positive and significant across almost all specifications. Starting from the single variable model in column (1), we progressively add controls and fixed effects: column (2) controls for the real GDP to capture the business cycle's effect on the advertising market. Starting from column (3), we report estimates for a fixed-effect model where the 
units of observations are the single channels. From column (4), we introduce time dummies, while in columns (5) and (6) we add channel-time controls: the channel's share of revenues in its segment and its rating. Since we only have US data, the real GDP control is dropped whenever time controls are included. All regressions are estimated with robust standard errors.

Table 2: Number of Incumbents and Avails - Effect by Segment

\begin{tabular}{|c|c|c|c|c|c|c|}
\hline & $(1)$ & $(2)$ & $(3)$ & $(4)$ & $(5)$ & $(6)$ \\
\hline Outlets & $\begin{array}{c}0.00615^{* * *} \\
(0.00164)\end{array}$ & $\begin{array}{l}0.00139 \\
(0.00496)\end{array}$ & $\begin{array}{c}0.00648^{* *} \\
(0.00104)\end{array}$ & $\begin{array}{c}0.00672^{* *} \\
(0.000878)\end{array}$ & $\begin{array}{c}0.00676^{* * *} \\
(0.000517)\end{array}$ & $\begin{array}{l}0.00160^{*} \\
(0.000459)\end{array}$ \\
\hline Outlets $\times$ Movies\&Series & $\begin{array}{c}-0.00613 \\
(0.00379)\end{array}$ & $\begin{array}{l}-0.0137^{*} \\
(0.00705)\end{array}$ & $\begin{array}{c}-0.00902^{* *} \\
(0.00164)\end{array}$ & $\begin{array}{c}-0.00811^{* *} \\
(0.00161)\end{array}$ & $\begin{array}{c}-0.00788^{* *} \\
(0.00177)\end{array}$ & $\begin{array}{c}-0.0137 * * * \\
(0.000325)\end{array}$ \\
\hline Outlets $\times$ Sports & $\begin{array}{r}-0.00261 \\
(0.00600)\end{array}$ & $\begin{array}{c}-0.00692 \\
(0.00700)\end{array}$ & $\begin{array}{c}-0.00687^{* *} \\
(0.000913)\end{array}$ & $\begin{array}{c}-0.00619^{* * *} \\
(0.000377)\end{array}$ & $\begin{array}{r}-0.00547 \\
(0.00200)\end{array}$ & $\begin{array}{c}-0.0154^{* * *} \\
(0.000117)\end{array}$ \\
\hline Real GDP & & $\begin{array}{l}0.00283 \\
(0.00247)\end{array}$ & $\begin{array}{l}0.00188^{*} \\
(0.000620)\end{array}$ & & & \\
\hline MoviesSeries_dummy & $\begin{array}{c}0.191^{* * *} \\
(0.0595)\end{array}$ & $\begin{array}{c}0.257^{* * *} \\
(0.0766)\end{array}$ & & & & \\
\hline Sports_dummy & $\begin{array}{c}0.106 \\
(0.0690)\end{array}$ & $\begin{array}{l}0.0493 \\
(0.0866)\end{array}$ & & & & \\
\hline Rev Mkt Share & & & & & $\begin{array}{l}0.156 \\
(0.398)\end{array}$ & \\
\hline Rating & & & & & & $\begin{array}{l}-0.0904 \\
(0.0542)\end{array}$ \\
\hline Constant & $\begin{array}{c}2.908 * * * \\
(0.0351)\end{array}$ & $\begin{array}{c}2.685^{* * *} \\
(0.190)\end{array}$ & $\begin{array}{c}2.760^{* * *} \\
(0.0469)\end{array}$ & $\begin{array}{c}3.242^{* * *} \\
(0.0372)\end{array}$ & $\begin{array}{c}3.226^{* * *} \\
(0.0621)\end{array}$ & $\begin{array}{c}3.273^{* * *} \\
(0.0258)\end{array}$ \\
\hline Observations & 416 & 416 & 416 & 416 & 415 & 279 \\
\hline$R^{2}$ & 0.048 & 0.050 & 0.284 & 0.307 & 0.311 & 0.299 \\
\hline Channel FE & NO & $\mathrm{NO}$ & YES & YES & YES & YES \\
\hline Time FE & $\mathrm{NO}$ & $\mathrm{NO}$ & NO & YES & YES & YES \\
\hline No. of Outlets & 56 & 56 & 56 & 56 & 56 & 33 \\
\hline
\end{tabular}

Table 2 reports the estimation results when we allow for heterogeneous effects in the number of channels across segments. The coefficients of interest are $\beta_{S}$ and $\beta_{M}$. Given our theory and our assumption that preferences are correlated within segments, we expect these coefficients to be negative. That is, we expect the effect of entry within the Sports and Movies\&Series segment to be diminished compared to the average industry effect (and possibly negative overall). Indeed, the coefficients have the expected sign in all regressions: the effect of the number of channels on advertising levels is positive for channels in the reference group ( $\beta$ is again positive and significant), while it is significantly lower for channels in the other two segments. This additional negative effect is particularly strong for Movies\&Series where 
$\left|\beta_{M}\right|>|\beta|$ in almost all specifications. Standard errors are clustered at the segment level.

To summarize, we obtain evidence of a positive relationship between entry and advertising levels. We also find a systematically reduced impact of entry on advertising levels within the same market segments. Based on our theory, we speculate that this difference comes from viewers' tastes for content which induce a good deal of overlap among viewers of the channels belonging to each of these segments.

The regressions above have the advantage of pooling data on different channels without taking a stance on the time it takes for entry to impact the incumbent choices. However, this strategy does not allow for accounting for within-channel omitted variables that vary over time. These variables may also operate at the segment level. To account for this, and as an alternative way to address the same issues, we also estimate a model for entry episodes, where our sample is now reduced to the periods when a given segment experiences the entry of a new channel. We estimate the following model:

$$
\begin{aligned}
\Delta \log \left(\text { Avails }_{i t}\right)= & \beta+\beta_{M} * \text { MoviesSeries_dummy }+\beta_{S} * \text { Sports_dummy } \\
& +\gamma * x_{i t}+\delta_{t}+\epsilon_{i t}
\end{aligned}
$$

This model can be obtained by first differencing the previous model around the years when entry occurs. In fact, $\Delta \log \left(\right.$ Avails $\left._{i t}\right)=\log \left(\right.$ Avails $\left._{i t+1}\right)-\log \left(\right.$ Avails $\left._{i t-1}\right)$ and the effect of entry (changed number of incumbents) is captured by the constant terms. Channel fixed effects are now excluded (as they cancel out in taking first differences), but we keep time fixed effects and also add some channel controls. The constant $\beta$ measures the effect of entry on the reference group, while $\beta_{S}$ and $\beta_{M}$ measure the additional effect for the Sports and Movies\&Series segments, respectively. The estimates reported in Table 3 confirm our previous results: entry episodes are associated with an increase in the quantity of avails in the reference group, while the effect is lower in the Sports and Movies\&Series segments. Since there are half as many observations in this setup, the point estimates are less precisely estimated than in Tables 1 and 2. Furthermore, because here we are looking at the effect one year after entry $(t+1)$, the magnitude of the parameters is notably bigger. The point estimate of the percent variation in avails due to an additional channel is on the order of $5 \%$ in column (3). Notably, the interaction term that captures the differential impact of entry in sports is around $11 \%$ less than the industry average. The difference is statistically and economically significant. 
Table 3: Entry Episodes - Average Effect and Effect by Segment

\begin{tabular}{lcccc}
\hline & $(1)$ & $(2)$ & $(3)$ & $(4)$ \\
\hline \multirow{2}{*}{ MoviesSeries dummy } & & $-0.0327^{* * *}$ & $-0.0494^{* *}$ & -0.0314 \\
& & $(0.000919)$ & $(0.00841)$ & $(0.0124)$ \\
Sports dummy & & $-0.0563^{* * *}$ & $-0.110^{* * *}$ & -0.0172 \\
& & $(0.000457)$ & $(0.00460)$ & $(0.0222)$ \\
$\Delta$ GDP [t-1,t+1] & 0.00380 & $0.00328^{*}$ & & \\
& $(0.00133)$ & $(0.00103)$ & & \\
Rating & & & $-0.0392^{* * *}$ & \\
& & & $(0.00128)$ & \\
Rev Mkt Share & & & & -0.171 \\
& & & & $(0.0987)$ \\
Constant & & & & \\
& 0.00393 & 0.0178 & $0.0615^{* *}$ & $0.263^{* * *}$ \\
& $(0.0213)$ & $(0.00901)$ & $(0.00875)$ & $(0.000141)$ \\
Observations & & & & \\
$R^{2}$ & 219 & 219 & 158 & 219 \\
Time FE & 0.009 & 0.028 & 0.121 & 0.091 \\
& $\mathrm{NO}$ & $\mathrm{NO}$ & YES & YES \\
\hline
\end{tabular}

Robust standard errors in parentheses

$$
\text { *** } \mathrm{p}<0.01, * * \mathrm{p}<0.05, * \mathrm{p}<0.1
$$

\subsection{Google Display Network}

\section{The GDN Exclusively Reaches $30 \%$ of Auto Insurance Seekers}

\begin{tabular}{|c|c|}
\hline \multicolumn{2}{|c|}{$\begin{array}{l}\text { \% Auto Insurance Seekers reached exclusively through } \\
\text { and NOT through... }\end{array}$} \\
\hline Yahoo! & $30 \%$ \\
\hline $\mathrm{AOL}$ & $66 \%$ \\
\hline MSN & $61 \%$ \\
\hline You Tube & $36 \%$ \\
\hline ESPN & $72 \%$ \\
\hline
\end{tabular}

\section{$30 \%$ of all Auto Insurance Seekers would not be reached through the Yahoo!}

Homepage but would be reached via the Google Display Network each day

\section{Key Takeaways}

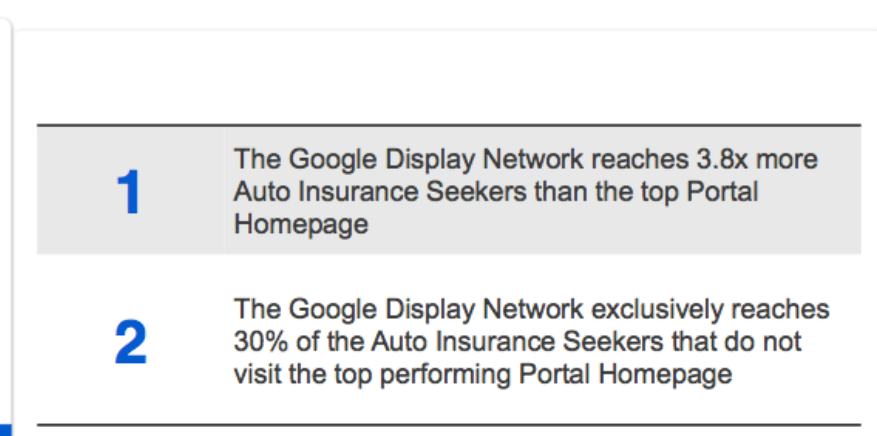

Figure 2: Exclusive GDN consumers in the Auto-Insurance market (2011)

Source: Excerpt from the Google's Reasearch study, "Google Display Network vs. Portal Takeovers for Auto Insurance seekers' available at http://www.google.com/think/research-studies/google-displaynetwork-vs-portal-takeovers-for-auto-insurance-seekers.html' (Last accessed 5/17/2013) 


\section{References}

[1] Ambrus, A. and R. Argenziano (2009): "Asymmetric networks in two-sided markets," American Economic Journal - Microeconomics, 1, 17-52.

[2] Anderson, S.P. and S. Coate (2005): "Market Provision of Broadcasting: A Welfare Analysis," Review of Economic Studies, 72, 947-972.

[3] Anderson, S.P. and Ø. Foros, and H.J. Kind (2014): "Competition for Advertisers and for Viewers in Media Markets,"Working Paper, University of Virginia and NHH Bergen.

[4] Anderson, S.P. and and M. Peitz (2012): "Advertising Congestion in Media Markets,"Working Paper, University of Virginia and University of Mannheim.

[5] Armstrong, M. (1999): "Competition in the Pay-TV Market," Journal of the Japanese and International Economies, 13, 257-280.

[6] Armstrong, M. (2006): "Competition in Two-Sided-Markets," RAND Journal of Economics, 37, 668-691.

[7] Athey, S., E. Calvano and J.S. Gans (2013): "The Impact of the Internet on Advertising Markets for News Media,"Working Paper, Harvard University.

[8] Bergemann, D. and A. Bonatti (2011): "Targeting in advertising markets: implications for offline versus online media," RAND Journal of Economics, 42, 417-443.

[9] Bernheim, B.D., and M.D. Whinston (1986): "Common Agency," Econometrica, 54, 923-942.

[10] Berry, S., and P. Reiss (2007): "Empirical Models of Entry and Market Structure,"in: Armstrong, M., and R. Porter (eds.) Handbook of Industrial Organization, vol. 3, Elsevier, Amsterdam, NorthHolland, 1845-1886.

[11] Butters, G.R. (1977): "Equilibrium Distributions of Sales and Advertising Prices," Review of Economic Studies, 44, 465-491.

[12] Clarke, E. (1971): "Multipart Pricing of Public Goods," Public Choice, 11, 17-33.

[13] Crampes, C., C. Haritchabalet, and B. Jullien (2009): "Advertising, Competition, and Entry in Media Industries," Journal of Industrial Economics, 57, 7-31.

[14] d'Aspremont, C., J.J Gabszewicz and J.-F. Thisse (1979): "On Hotelling's "Stability in Competition","Econometrica, 47, 1145-1150.

[15] Dukes, A. and E. Gal-Or (2003): "Negotiations and Exclusivity Contracts for Advertising," Marketing Science, 22, 222-245.

[16] Ellison, G. and D. Fudenberg (2003): "Knife Edge or Plateau: When Do Market Models Tip?," Quarterly Journal of Economics, 118, 124-1278.

[17] Fisher, F.M., J.J. McGowan and D.S. Evans (1980): "The Audience-Revenue Relationship for Local Television Stations," Bell Journal of Economics, 11, 694-708.

[18] Gentzkow, M., J.M. Shapiro, and M. Sinkinson (2014): "Competition and Ideological Diversity: Evidence from US Newspapers," American Economic Review, forthcoming. 
[19] George, L. and J. Waldfogel (2003): "Who Affects Whom in Daily Newspaper Markets?," Journal of Political Economy, 11,765-785.

[20] Harbord, D. and M. Ottaviani (2011): “Contract and Competition in the Pay-TV Market,"Working Paper, Bocconi University.

[21] Groves, T. (1973): "Incentives in Teams," Econometrica, 41, 617-631.

[22] Kind, H.J., T. Nilssen, and L. Sørgard (2009): "Business Models for Media Firms: Does Competition Matter for how they Raise Revenue?," Marketing Science, 28, 1112-1128.

[23] Kreps, D.M. and J.A. Scheinkman (1983): "Quantity Precommitment and Bertrand Competition yield Cournot Outcomes," Bell Journal of Economics, 14, 326-337.

[24] Martimort, D. and L. Stole (2009): "Market Participation in Delegated and Intrinsic CommonAgency Games," RAND Journal of Economics, 40, 78-102.

[25] Ozga, S.A. (1960): "Imperfect Markets through Lack of Knowledge," Quarterly Journal of Economics, 74, 29-52.

[26] Peitz, M. and T. Valletti (2008): "Content and Advertising in the Media: Pay-TV versus Free-toAir," International Journal of Industrial Organization, 26, 949-965.

[27] Rochet, J.-C. and J. Tirole (2003): "Platform Competition in Two-Sided-Markets," Journal of the European Economic Association, 1, 990-1029.

[28] Rüdiger, J. (2013): “Cross-Checking the Media,"Working Paper, European University Institute Florence.

[29] Shaked, A. and J. Sutton (1977): "Relaxing Price Competition through Product Differentiation," Review of Economic Studies, 49, 3-13.

[30] Spence, M. and B. Owen (1977): "Television Programming, Monopolistic Competition, and Welfare," Quarterly Journal of Economics, 91, 103-126.

[31] Steiner, P.O. (1952): "Program Patterns and the Workability of Competition in Radio Broadcasting," Quarterly Journal of Economics,, 66, 194-223.

[32] Strömberg, D. (2004): "Mass Media Competition, Political Competition, Public Policy," Review of Economic Studies, , 71, 265-284.

[33] Sweeting, A. (2013): "Dynamic Product Positioning in Differentiated Product Markets: The Effect of Fees for Musical Performance Rights on the Commercial Radio Industry," Econometrica, 81, 17631803.

[34] Vives, X. (2000): Oligopoly Pricing: Old Ideas and New Tools, MIT Press: Cambridge, Massachusetts.

[35] Wildman, S.S. and B. Owen (1985): "Program Competition, Diversity, and Multi-Channel Bundling in the New Video Industry,"in: E. Noam (ed.), Video Media Competition: Regulation, Economics and Technology, Columbia University Press: New York, 244-273. 